\title{
AGN Obscuration and the Unified Model
}

\author{
Stefano Bianchi, ${ }^{1}$ Roberto Maiolino, ${ }^{2,3}$ and Guido Risaliti ${ }^{4,5}$ \\ ${ }^{1}$ Dipartimento di Fisica, Università degli Studi Roma Tre, Via della Vasca Navale 84, 00146 Roma, Italy \\ ${ }^{2}$ INAF-Osservatorio Astronomico di Roma, Via di Frascati 33, 00040 Monte Porzio Catone, Italy \\ ${ }^{3}$ Cavendish Laboratory, University of Cambridge, 19 J. J. Thomson Avenue, Cambridge CB3 OHE, UK \\ ${ }^{4}$ INAF-Osservatorio Astrofisico di Arcetri, L.go E. Fermi 5, Firenze, Italy \\ ${ }^{5}$ High Energy Astrophysics Division, Harvard-Smithsonian Center for Astrophysics, 60 Garden Street, Cambridge, MA 0218, USA
}

Correspondence should be addressed to Stefano Bianchi, bianchi@fis.uniroma3.it

Received 31 August 2011; Accepted 29 December 2011

Academic Editor: Isabelle Gavignaud

Copyright (๑) 2012 Stefano Bianchi et al. This is an open access article distributed under the Creative Commons Attribution License, which permits unrestricted use, distribution, and reproduction in any medium, provided the original work is properly cited.

Unification Models of Active Galactic Nuclei postulate that all the observed differences between type 1 and type 2 objects are due to orientation effects with respect to the line of sight to the observer. The key ingredient of these models is the obscuring medium, historically envisaged as a toroidal structure on a parsec scale. However, many results obtained in the last few years are clearly showing the need for a more complex geometrical distribution of the absorbing media. In this paper, we review the various pieces of evidence for obscuring media on different scales, from the vicinity of the black hole to the host galaxy, in order to picture an updated unification scenario explaining the complex observed phenomenology. We conclude by mentioning some of the open issues.

\section{Introduction: The Standard Unified Model}

In this paper, we discuss the recent developments on the AGN unified models, specifically for what concern the geometry, location, and physics of the absorbing medium. Before discussing the more recent results, in this section we shortly review the early, classical arguments that historically led to the formulation of the standard Unified Model. An early review of the initial results was also given in Antonucci [1].

The first unification attempts have been focussed on polarization measurements. In particular Antonucci [2] found a perpendicular alignment of optical polarization relative to the radio axis in a sample of radio galaxies, which was interpreted as due to scattering of photons, whose direction before entering the line of sight was primarily in the vertical direction. Shortly after, additional evidence was found in low luminosity, local AGN, and specifically Seyfert galaxies [3]. Seyfert 1 galaxies are characterized by the presence of broad optical permitted lines (FWHM > $1000 \mathrm{~km} / \mathrm{s}$ ), such as $\mathrm{H} \alpha$ and $\mathrm{H} \beta$, that are not observed in Seyfert 2 galaxies. However, the presence of both strong high ionization and low ionization narrow (FWHM $<1000 \mathrm{~km} / \mathrm{s}$ ) forbidden lines (such as [O III], [Ne III], [O II], [O I], [N II], [S II]), and several very high ionization coronal lines (such as [Fe X], [Fe XI], [Si IX], [Si X]) is common to both types of Seyfert galaxies and with similar line ratios. The latter finding suggested that all Seyfert galaxies are powered by the same intrinsic engine.

A strong observational evidence of a unification between type 2 and type 1 Seyfert nuclei has been the discovery of broad optical lines in the polarized spectrum of the archetypal Seyfert 2, NGC 1068, obtained by Antonucci and Miller [4]. This finding revealed the presence of a Broad Line Region (BLR) in this Seyfert 2 nucleus, which is hidden to our line of sight, but whose light is scattered in our direction from material (in the case of NGC 1068, likely free electrons in ionized gas) distributed on scales larger than the absorber. Such reflected light is very weak compared to the light of the galaxy, hence difficult to detect in the total spectrum, but it is highly polarized, and therefore detectable in the polarized spectrum. The basic idea of the Unified Model is that type 2 and type 1 AGN are intrinsically the same class of objects and their differences are only due to orientation effects relative to an obscuring medium. 
The need for a "toroidal", axisymmetric structure of the absorber was initially inferred from the fact itself that the reflected broad lines are polarized and from the measured polarization angles. If the absorber was a simple cloud along the line of sight, then reflection should come from all directions, hence by averaging from all angles the total polarization should be zero. In order to break the symmetry of the polarization angles the absorber should prevent the nuclear light to be scattered in a significant range of angles, and a "torus" is the most natural configuration that can achieve this effect.

The size of the toroidal absorber was initially postulated to be on the parsec scale $[5,6]$. Such typical size was simply inferred by the need for the absorber to be large enough to obscure the BLR, which in Seyfert nuclei has a size well below a parsec, based on reverberation studies (e.g., [7, 8]), but small enough not to obscure the Narrow Line Region (NLR), which is distributed on the $10-100$ pc scale. However, as we will discuss extensively in this paper, there is clear evidence that the absorbing medium is also distributed on smaller and larger scales.

Since the seminal discovery of Antonucci and Miller [4], polarized broad lines have been discovered in several other Seyfert 2 nuclei $[9,10]$, contributing to the generalization of the unified model to the whole class of type 2 AGN. Further evidence for a unified theory between type 2 and type 1 AGN has been obtained from various multiwavelength studies. In a number of type $2 \mathrm{AGN}$ the presence of an obscured BLR was inferred from the detection of a broad component of hydrogen recombination lines in the near infrared, such as $\operatorname{Pa} \beta, \operatorname{Pa} \alpha, \operatorname{Br} \gamma$, where dust absorption can be several times lower than in the optical [11-15].

Hard X-ray observations of AGN have provided additional, unambiguous evidence in favor of the unified model, obtained already with the early hard X-ray satellites such as Exosat, Ginga, ASCA, and BeppoSAX [16-19]. Although generally weak or even undetected in the soft X-ray band $(<2 \mathrm{keV})$, most type $2 \mathrm{AGN}$ are detected in the hard $\mathrm{X}$ rays $(>2 \mathrm{keV})$ and are characterized by a power-law spectrum similar to Syls, favoring a common central engine, but affected by a photoelectric absorption cutoff directly demonstrating the presence of an absorbing medium along the line of sight, with a column density typically well in excess of $10^{22} \mathrm{~cm}^{-2}$. In a number of Seyfert $2 \mathrm{~s}$ the hard Xray spectrum does not show the presence of a prominent absorption cutoff, but it is characterized by a very strong $\mathrm{Fe} \mathrm{K} \alpha$ line at $6.4 \mathrm{keV}$, with an equivalent width larger than $500 \mathrm{eV}$ (e.g., [19-21]). Such high equivalent widths of the Fe line can only be explained by assuming that the direct Xray radiation is totally absorbed by a Compton thick medium $\left(\mathrm{N}_{\mathrm{H}}>10^{24} \mathrm{~cm}^{-2}\right)$ and that the observed (weak) continuum and $\mathrm{Fe} \mathrm{K} \alpha$ are due to reflection from the circumnuclear medium. A detailed discussion of these effects and on the distribution of the reflecting medium will be given in the next sections.

If the NLR in type 2 AGN is created by the photoionization of a nuclear UV/X source hidden from our line of sight by a toroidal absorber, then the expectation is that the NLR should have a (bi)conical morphology, due to the light cones defined by the nuclear absorber. High-resolution, narrow band imaging (or integral field spectroscopy), especially with the advent of HST, has indeed revealed such ionization cones on scales ranging from a few $10 \mathrm{pc}$ up to several $100 \mathrm{pc}$, in many nearby AGN (e.g., [22-25]). The opening angle of the cones gives the fraction of the sky hidden to our line of sight, which is in reasonable agreement with what inferred from the relative fraction between type 1 and type 2 AGN in the local universe [26]. It is interesting that the axis of the ionization cones is often not aligned with the minor axis of the host galaxy, meaning that the circumnuclear absorber is not necessarily aligned with the gaseous disk of the host galaxy. Generally the orientation of the ionization cone axis is in the same direction as the radio jet, but often the two are not exactly aligned, meaning that even on small (parsec or subparsec) scales there is a slight misalignment between the dusty absorbing medium and the central engine (the accretion disc) $[24,27]$.

We conclude this introduction by shortly mentioning that several theoretical works have modelled the physics and the structure of the "toroidal" absorbing medium in the attempt of reproducing the observable properties. Initial models had assumed a simple toroidal structure with a uniform distribution of gas and dust with a parsecscale radius (e.g., [6, 28]), while other models suggested more extended geometries, up to $100 \mathrm{pc}$, to explain the broad infrared spectral energy distribution observed in AGN [29]. One of the main problems of these models is their dynamical stability. Often radiation pressure from infrared photons within the torus is invoked as a solution to keep the torus geometrically thick [30]. Other authors ascribe the geometrical thickness of the torus to turbulence introduced by supernovae or stellar winds [31, 32]. It has also been proposed that a wide angle of obscuration does not necessarily require a geometrically thick torus, but can also be achieved with a warped or tilted disk [33-36].

Recently, the hypothesis of uniform gas and dust distribution has been abandoned by many models, by introducing a clumpy structure of the absorbing medium [37-40]. These models can account for several of the observational properties of AGN and, most importantly, are strongly supported by recent X-ray observations that directly reveal the clumpiness of the absorbing medium, as discussed in the following sections.

\section{From Galactic to Sub-Pc Scale: Absorption at Different Scales}

One of the most significant new aspects on the structure of AGN, as emerged in last few years, is that the standard, parsec-scale "torus" is not enough to explain all the complex absorption features discovered by many observations. While the unified picture remains valid in its more general sense (i.e., the presence of nonspherically symmetric absorbers at the origin of the type 1/type 2 dichotomy) several new observations and models, mostly in the X-ray and infrared domain, suggest that multiple absorbers are present around the central source, on quite different physical scales. In the 
following we review the main observational evidence for each of them, together with some brief discussion on their physical interpretation.

2.1. Absorption within the Sublimation Radius. The evidence for gas absorption within the sublimation radius comes mostly from X-ray observations. The most direct way to probe the presence of such gas component is through absorption variability measurements.

$\mathrm{X}$-ray absorption variability is a common feature in AGN. An analysis of a sample of nearby obscured AGN with multiple $\mathrm{X}$-ray observations, performed a few years ago [41] revealed that column density $\left(\mathrm{N}_{\mathrm{H}}\right)$ variations are almost ubiquitous in local Seyfert galaxies. More recent observations performed with XMM-Newton, Chandra, and Suzaku further confirmed this finding. The physical implications of these measurements are that the circumnuclear X-ray absorber (or, at least, one component of it) must be clumpy and located at subparsec distances from the central source.

The comparison between different observations, typically performed at time distances of months-years, only provides upper limits to the intrinsic time scales of $\mathrm{N}_{\mathrm{H}}$ variations. An improvement of these estimates could only be obtained through observational campaigns within a few weeks/days, and/or through the search for $\mathrm{N}_{\mathrm{H}}$ variations within single long observations. Such short time-scale studies have been performed for a handful sources: NGC 1365 [42-45], NGC 4388 [46], NGC 4151 [47], NGC 7582 [48], and Mrk 766 [49].

In particular, in the case of NGC 1365, Chandra, XMMNewton, and Suzaku observations revealed extreme spectral changes, from Compton thin $\left(\mathrm{N}_{\mathrm{H}}\right.$ in the range $\left.10^{23} \mathrm{~cm}^{-2}\right)$ to reflection dominated $\left(\mathrm{N}_{\mathrm{H}}>10^{24} \mathrm{~cm}^{-2}\right)$ on time scales from a couple of days to $\sim 10$ hours (see Figure 1). Such rapid events imply that the absorption is due to clouds with velocity $v>10^{3} \mathrm{~km} \mathrm{~s}^{-1}$, at distances from the $\mathrm{BH}$ of the order of $10^{4}$ gravitational radii (assuming that they are moving with Keplerian velocity around the central black hole). The physical size and density of the clouds are estimated to be of the order of $10^{13} \mathrm{~cm}$ and $10^{10}-10^{11} \mathrm{~cm}^{-3}$, respectively. All these physical parameters are typical of BLR clouds, strongly suggesting that the $\mathrm{X}$-ray absorber and the clouds responsible for broad emission lines in the optical/UV are one and the same.

These results are obtained from the analysis of the observed absorption variations in the sources mentioned above, assuming a simple scheme, where the clouds are homogeneous, with a constant column density and are moving across the line of sight with Keplerian velocity. However, in a few cases with particularly high signal-to-noise ratio, the analysis of X-ray "eclipses" can provide further information on the geometrical and physical structure of the cloud.

In the case of NGC 1365, a careful analysis [45] of the spectral X-ray variability during two eclipses revealed a "cometary" shape of the obscuring cloud, consisting of a high-density head, and an elongated, lower-density tail. This structure is revealed by the time evolution of the two key observational parameters of the cloud (Figure 2): its covering factor to the X-ray source (suddenly increasing at the beginning of the occultation, then slowly increasing over a relatively long time interval), and its column density (highest at the beginning of the occultation, and then decreasing steadily).

Occultations have also been observed in the bright Narrow Line Seyfert 1 Mrk 766 [49], revealing that such events are possible (though rare) in on-average unobscured sources. While the data quality was not enough to perform a column density/covering factor deconvolution as the one described above for NGC 1365, additional information came from the detection of highly ionized iron absorption lines. These lines, due to Fe XXV and Fe XXVI ions, are present only in the same time intervals where the occultations due to the neutral cloud are also present. Furthermore, their energy clearly reveal that the absorbing gas is outflowing with velocities of several $10^{3} \mathrm{~km} \mathrm{~s}^{-1}$. The straightforward interpretation of these measurements is that the absorption is due to an outflowing cloud, with a high-density, lowionization head, and a low-density, high-ionization tail.

The above examples remain unique among the available $\mathrm{X}$-ray observations of AGN, but they suggest that with future, larger area X-ray observatories, $\mathrm{X}$-ray absorption variability may become a powerful, relatively standard way to directly measure the physical properties of the absorbing BLR clouds.

One of the most direct consequences of the presence of gas inside the dust sublimation radius is a decrease of the expected dust-to-gas ratio, as measured from the ratio between optical/near-IR reddening, and X-ray column density. This is a well-known observational evidence in nearby Seyfert galaxies (e.g., $[50,51]$ ), which is therefore naturally explained in the context of X-ray absorption by BLR clouds.

The presence and structure of "cometary" clouds poses challenges and possible solutions for long-standing problems in modeling the BLR in AGN. Maiolino et al. [45] estimate that the cloud head loses a significant fraction of its mass through the cometary tail, which is expected to cause the total cloud destruction within a few months. If these clouds are representative of most BLR clouds (or at least the highionization ones), this implies that the BLR region must be continuously replenished with gas clouds, possibly from the accretion disk. Such a "dynamical equilibrium" scenario would solve the problem of the long-term stability of BLR clouds, for which a convincing solution has not been found yet.

A further possible interesting aspect of the "cometary" structure (not yet explored in the literature) is the reduction of the number of clouds needed to explain the smoothness of the profiles of the broad absorption lines. High-quality observations of the profile of optical broad emission lines suggest that the minimum number of clouds needed to reproduce the observed smoothness is quite high: one of the most striking examples is that of NGC 4151, with an estimated number of at least $10^{8}$, based on high S/N Keck spectra [52]. Considering that the estimated black hole mass of NGC 4151 is of the order of $10^{7} \mathrm{M}_{\odot}$, and that the distance of BLR clouds is of the order of $10^{4}$ gravitational radii, a huge 


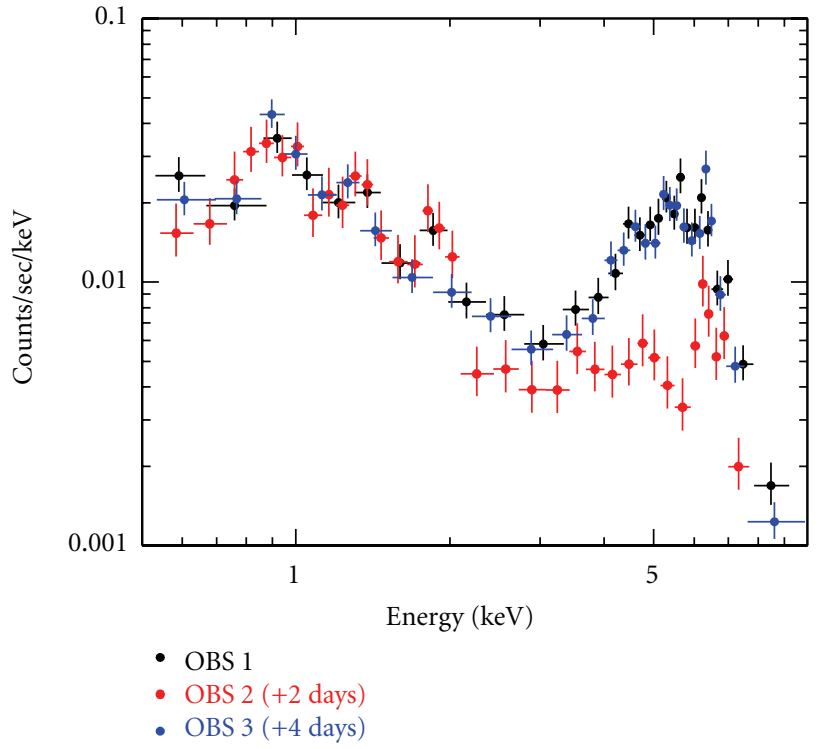

(a)

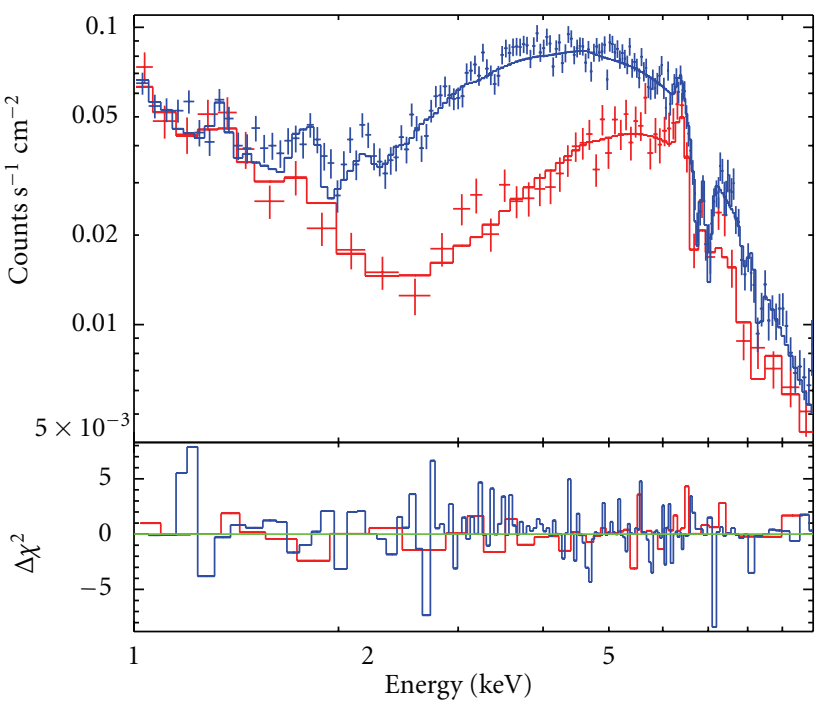

(b)

Figure 1: Spectra of NGC 1365, showing X-ray occultations due to BLR clouds: (a) spectra obtained from three Chandra snapshot observations performed every two days [43]. The reflection-dominated spectrum in the second observation is due to an occultation by a Compton-thick cloud (the only one ever detected in short time scales). (b) Spectra obtained from different intervals (length of about 10ks) of a single Suzaku observation, revealing absorption changes due to a cloud with a column density of the order of $10^{23} \mathrm{~cm}^{-2}$.

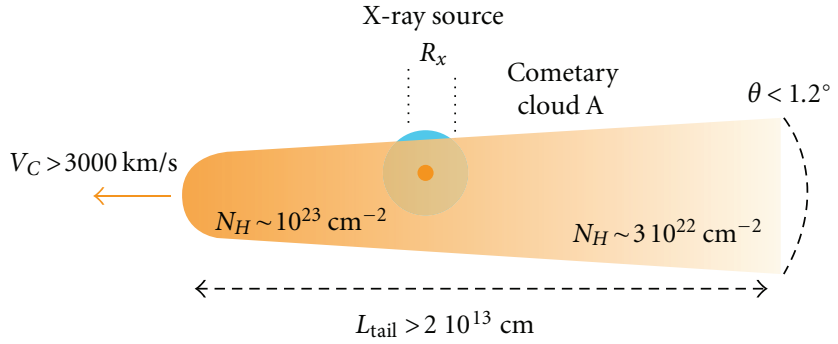

FIGURE 2: Structure of the absorbing cloud as obtained from a Suzaku observation of NGC 1365. The estimates are based on the hypothesis of Keplerian motion, and on a black hole mass of $2 \times 10^{6} \mathrm{M}_{\odot}[45]$. The cloud size is not in the correct scale: the tail is much longer when compared with the source size, which is of the order of a few $10^{11} \mathrm{~cm}$.

cloud density is obtained, with a nearly complete occupation of the available volume. If instead every single cloud has a cometary structure, analogous to that inferred for NGC 1365 , each cloud would contribute to the observed profile with a small, but not null, width, which would greatly reduce the total number of clouds in order to reproduce the same smoothness.

Finally, if the covering factor and the optical depth of the BLR are large enough, a significant fraction of the iron $\mathrm{K} \alpha$ line should be produced there. In order to test this hypothesis, NGC 7213 represents a unique opportunity. The $\mathrm{X}$-ray spectrum of this source has no evidence for Compton reflection, a unique result among bright Seyfert 1s [53-55]. The observed neutral iron $\mathrm{K} \alpha$ line, therefore, cannot be produced in a Compton-thick material, like the disc or the torus. Indeed, the iron line is resolved in a Chandra High-Energy Transmission Grating observation, with a measured FWHM which is in perfect agreement with the value measured for the broad component of the $\mathrm{H} \alpha$ in a simultaneous optical observation [56]. Moreover, the observed equivalent width (EW) of the iron line is in agreement with an origin in the BLR, under reasonable assumptions on the geometrical distribution of the clouds, their covering factor, and their column density [56]. Therefore, NGC 7213 is the only Seyfert 1 galaxy whose iron $\mathrm{K} \alpha$ line is unambiguously produced in the BLR. It is difficult to reach similar conclusions for other objects, because of the presence of the Compton reflection component never allows us to exclude the contribution, from the more extended torus, and because it is difficult to measure the FWHM of the iron line and compare it with the optical lines (see also next section). In the future, highresolution X-ray spectroscopy with microcalorimeters and $\mathrm{X}$-ray reverberation studies will be extremely powerful in tackling this issue. 
2.2. Absorption form Pc-Scale Tori. Early evidence for a circumnuclear dusty medium on parsec, or subparsec scales, as initially invoked by the Unified Model, was obtained from near-IR studies, which revealed the presence of very hot dust, close to the sublimation temperature, in the nuclei of Syls [57-59]. The dust sublimation radius in Seyfert nuclei is on subparsec scales and on parsec scales at quasar luminosities. The subparsec location of the hot dust emitting in the near-IR has been confirmed by extensive reverberation observational campaigns [60], which also confirmed the expected $L^{1 / 2}$ dependence of the sublimation radius. The covering factor of the circumnuclear dusty medium, inferred from the near-IR observations, is very high in most of the nearby Syls (exceeding 0.8: [61, 62]), and generally in agreement with the observed type 2/type 1 ratio [26].

Radio VLBI observations were the first ones to effectively image the AGN circumunclear medium on parsec and subparsec scales. Greenhill et al. [63] obtained VLBA water maser images at subparsec resolution of NGC 1068, revealing a rotating warped disk structure. The warping of the maser disk orientation may indicate that the inclination of the nuclear molecular disk is likely responsible for the large covering faction, rather than a geometrically thick torus [36]; however, one must also take into account that the maser emission does not necessarily trace the global morphology of the circumnuclear molecular gas, but only the equatorial edge-on medium (within $\sim 15^{\circ}$ from the line of sight) where maser amplification is highest. VLBI water maser observations were subsequently obtained for other AGN, finding similar structures [64-67]. Gallimore et al. [68] obtained VLBA images of the nuclear continuum radio emission of NGC 1068. Beside the nuclear nonthermal emission, tracing the nuclear engine, they resolved two symmetric radio free-free blobs at a radius of $\sim 0.3 \mathrm{pc}$, which were interpreted as the inner ionized edge of the obscuring torus.

The possibility to effectively "image" the dusty component of the parsec-scale torus has become possible in 2004, when mid-infrared interferometry allowed Jaffe et al. [69] to map for the first time the dust at parsec resolution in the Seyfert 2, NGC 1068. Their results, refined by following observations [70], are consistent with a two-component dust distribution: an inner ( $0.5 \mathrm{pc}$ of thickness), rather elongated hot $(T>800 \mathrm{~K})$ component, and a more extended (3-4 pc), less elongated colder $(T \simeq 300 \mathrm{~K})$ component. The compact component is coincident, in size and orientation, with the nuclear water maser. Most of the absorption appears to be located outside $1 \mathrm{pc}$. A similar result was found, with the same technique, for another Seyfert 2, Circinus: again two components, an inner and more compact $(0.4 \mathrm{pc})$, and an outer $(2 \mathrm{pc})$ component [71]. However, the temperature of the inner component in Circinus $(T=330 \mathrm{~K})$ is significantly lower than in NGC 1068, and far from the sublimation temperature (see Figure 3). The first observation carried on a type 1 object, NGC 4151, led to results in agreement with the previous ones on Seyfert 2s [72].

When such interferometric studies were performed on a sizeable sample of objects in mid- and near-infrared, it was observed that no significant differences are found between type 1 and 2 sources and the size of the dusty emitter scales with the square root of the luminosity [73-75]. Comparisons with tori models suggest that, in principle, it would be possible to disentangle between face-on and edgeon distributions by comparing the compactness of the dusty structure to the AGN luminosity, but uncertainties on the observed measures are still too large.

The presence of Compton-thick neutral material with large covering factor in the environment of AGN is also supported by the ubiquitous presence of the iron $\mathrm{K} \alpha$ line and the Compton reflection component in the X-ray spectra of Seyfert galaxies (e.g., $[54,76,77])$. Although a component broadened by strong gravity effects arising in the accretion disk is observed in at least a third of the sources (see, e.g., $[78,79])$, a narrow core of the iron line is a much more common feature. The line, typically unresolved (with upper limits of several thousand $\mathrm{km} / \mathrm{s}$ for its FWHM), must be produced far from the nucleus, either in the BLR, the torus, or the NLR. Apart from single exceptional cases (like NGC 7213: see previous section), current $\mathrm{X}$-ray satellites allow us to resolve its FWHM only in a few objects and with limited information, generally leading to inconclusive estimates on the location of the material producing the lines (see, e.g., $[80,81])$. Future $\mathrm{X}$-ray missions taking advantage of microcalorimeters will represent a breakthrough in this kind of analysis, allowing us to deconvolve all the components possibly present in the iron line, as routinely performed for the optical lines.

However, clues in favour of a parsec-scale distance of the material producing the narrow iron line and the Compton reflection component already come from the lack of variability of these features. X-ray spectra of Comptonthick sources (i.e., obscured by a column density larger than $\simeq 10^{24} \mathrm{~cm}^{-2}$ ) are completely dominated by reflection features, and they typically do not show any variability even on long time scales. This is particular clear in sources where the central engine fades away for a long time interval (years), while the reflection component (including the $\mathrm{Fe} \mathrm{K} \alpha$ line) remains stable over the same time scale $[82,83]$. This suggests that the obscuration/reflection occurs on (at least) pc-scale, like the standard torus envisaged in the Unification Models and mapped by interferometry. In principle, the geometry and distance of the torus could be estimated by doing accurate X-ray reverberation analysis of the iron line and the Compton reflection component, in order to take into account in detail how the material reacts to the intrinsic variability of the central source. Unfortunately, such a study is extremely difficult and uncertain with current Xray missions.

While X-ray absorption variability studies have delivered exquisite information on the structure of the absorbing medium on scales of the BLR, in particularly for what concerns the clumpiness of the absorber, the same kind of analysis is difficult to perform on the parsec scale absorber, both because the time scale for variation is much longer, and because it is totally overwhelmed by the variability introduced by the clouds in the BLR. However, as mentioned in the introduction, recent models have shown that even for the dusty pc-scale torus a clumpy structure (Figure 4) 


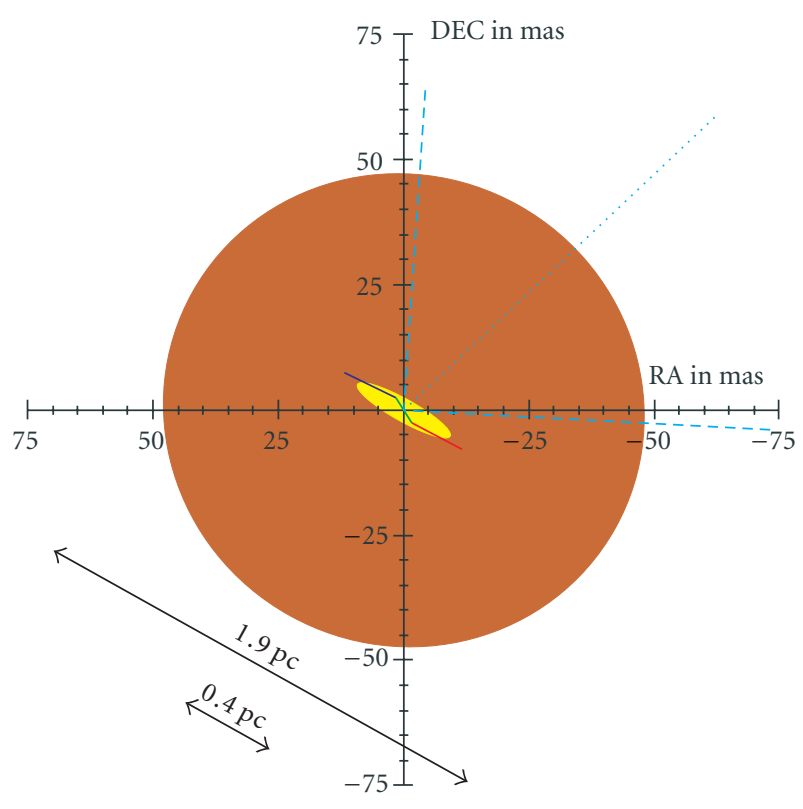

(a)

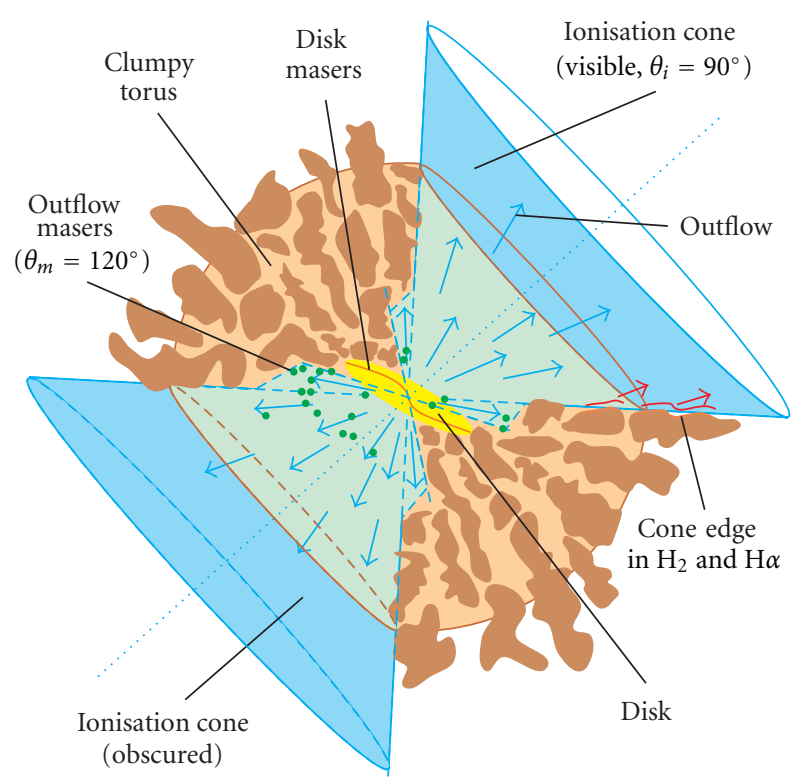

(b)

Figure 3: The Circinus Galaxy (from Tristram et al. [71]). (a): Sketch of the best-fit physical model where a warm ( $T \simeq 330 \mathrm{~K}$ ) emission region (yellow) is surrounded by a cooler $(T \simeq 300 \mathrm{~K}$ ) one (brown). The water maser emitters are over-plotted (blue and red lines), together with the ionised cone (dashed and dotted light blue line). (b): the dusty torus as derived from the MIR interferometric observations.

can better account for the infrared observational properties [38, 84-86]. In particular, the very broad infrared Spectral Energy Distribution (SED) of AGN requires dust at multiple temperature, which is hardly achieved by models with a compact (pc-scale) uniform torus [28]. Large-scale (100 pc) dusty torii [87] can reproduce the broad IR SED, but can be hardly reconciled with the small sizes observed in midIR interferometric observations. A pc-scale, but clumpy torus can at the same time match the observed mid-IR size and reproduce the broad range of dust temperatures, since within each dense clump dust does span a wide range of temperatures. A crucial test of these models will be feasible with $A L M A$, which will allow us to image the cooler dust thermal emission of torus at sub-mm wavelengths at subpc resolution. Indeed, as discussed in Maiolino [88], a clear prediction of this model is that the morphology of the "torus" at far-IR/sub-mm wavelengths (tracing cold dust) should be very similar to the morphology observed in the mid-IR (tracing warm dust). An independent observational indication of the clumpiness of the dusty absorber is, as discussed in Shi et al. [89] and in Nikutta et al. [90], the large scatter of the depth of the $9.7 \mu \mathrm{m}$ silicate absorption feature (directly tracing dust absorption at mid-IR wavelengths) as a function of the X-ray gaseous column density, as well as the finding that the same feature is observed in emission in some type 2 AGN.

2.3. Absorption by Gas in the Host Galaxy. Although clear evidence in favour of absorption from the BLR and the pcscale "torus" has been presented in the previous sections, in some cases the lowest column densities are consistent with

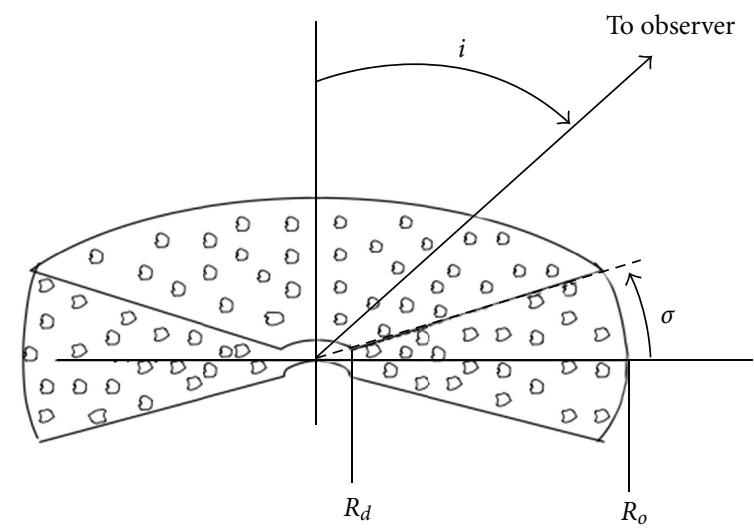

Figure 4: Sketch of the dusty torus as in the model of Nenkova et al. [38], which can reproduce the observed dust temperature distributions derived from infrared observations.

the optical reddening associated with the medium in the host galaxy, which is therefore an additional ingredient that must therefore be considered in a global Unification Model (see, e.g., $[26,91])$. Early evidence of obscuration on $100 \mathrm{pc}$ scales by the host galaxy gaseous disk came from the finding that optically selected AGN samples tend to avoid edge-on systems [26], a result which has been confirmed and refined with much higher statistics by using the SDSS survey [92]. Furthermore, it has been suggested that the gas in the host galaxy disk can partially obscure also the NLR.

Further direct evidence for obscuration on "large" scales was obtained through high-resolution HST images, showing 
that dust lanes at distances of hundreds of parsecs are very common in Seyfert galaxies [93]. The presence of these structures is correlated with Compton-thin X-ray obscuration, even if not necessarily being directly responsible of the obscuration of the nucleus [94]. In some cases, the effect of dust lanes can be seen directly as X-ray obscuration towards the soft X-ray emission from the NLR (e.g., [95]) see Figure 5.

Interferometric maps of the molecular gas distribution have provided additional evidence for large amount of dense gas on the $100 \mathrm{pc}$ scale surrounding AGN, which certainly contributes to the obscuration of the central engine along some line of sights (e.g., [96-98]). Certainly, within this context, the advent of ALMA is going to be a breakthrough by providing detailed maps of the molecular gas distribution in the circumnuclear region of many AGN.

As it will be discussed further below, there is evidence that the role of absorption from gas in the host galaxy becomes increasingly important in high-z AGN $[99,100]$, likely as a consequence of the higher gas content and higher star formation in high-z AGN hosts.

It is worth reminding that the obscuration occurring on such large scales is limited by dynamical mass constraints. Risaliti et al. [101] showed that Compton-thick gas must be contained on scales significantly smaller than $100 \mathrm{pc}$, in order not to exceed the dynamical mass in the same region and have a covering factor large enough to account for the high number of observed Compton-thick sources. This in turns means that the bulk of the ubiquitous Compton reflection component and narrow neutral iron $\mathrm{K} \alpha$ line must also come from a compact region. However, in NGC 1068 hard X-ray emission (mostly associated to the reflection component) and the neutral iron line are seen extending up to $\simeq 2 \mathrm{kpc}$ from the nucleus [102].

\section{Open Issues}

3.1. "True" Seyfert 2s. As discussed in Section 1, one of the most convincing pieces of evidence in favor of the Unification Model is the detection of broad optical lines in the polarized spectra of type 2 AGN. However, about half of the brightest Seyfert 2 galaxies appear not to have hidden broad-line regions in their optical spectra, even when highquality spectropolarimetric data are analysed $[9,10]$.

A number of these cases may be associated with nuclei where the mirror reflecting the broad lines either has very low scattering efficiency (either due to low covering factor or low column density) or is obscured [103]. Evidence was also obtained that the lack of polarized broad lines is associated with a stronger contribution/dilution from the host galaxy or from a circumnuclear starburst, making the detection of polarized broad lines harder [104, 105].

However, a number of Sy2s without polarized broad lines may be genuine type 2 Seyferts, in the sense that they intrinsically lack a BLR. Indeed, observational evidence suggests that Seyfert 2s with polarized broad lines are more easily associated with truly obscured Seyfert 1 nuclei, while Seyfert $2 \mathrm{~s}$ without polarized broad lines preferentially host weak AGN, possibly incapable of generating a classical BLR $[9,10]$.

In a model proposed by Nicastro [106], the creation of the BLR is connected with disk instabilities occurring in proximity of a transition radius at which the accretion disk changes from gas-pressure dominated to radiation-pressure dominated. Since this transition radius becomes smaller than the innermost stable orbit for very low accretion rates (and therefore luminosities), very weak AGN are expected not to be surrounded by a BLR. More recently, the disappearance of the BLR in low-luminosity AGN has been predicted by Elitzur and Ho [107] and Trump et al. [108] within a diskwind scenario, where the BLR is embedded in an outflowing wind, which is no longer supported by the disk below a critical value of the Eddington ratio.

If the BLR cannot form in weakly accreting AGN, we expect the existence of "true" Seyfert 2 galaxies, that is, optically classified Type 2 objects, without any evidence of obscuration of their nuclei. Such unabsorbed Seyfert 2 galaxies do exist, and the best examples (where the lack of the optical broad lines and of the X-ray obscuration are unambiguously found in simultaneous observations with high SNR) have low Eddington rates: NGC 3147 $\left(\log L_{\mathrm{bol}} / L_{\mathrm{Edd}} \simeq-4\right.$ : [109]), Q2131427 $\left(\log L_{\mathrm{bol}} / L_{\mathrm{Edd}} \simeq-2.6\right.$ : [110]), and NGC $3660\left(\log L_{\mathrm{bol}} / L_{\mathrm{Edd}} \simeq-2\right.$ : [111, 112]). When a sizeable catalogue of X-ray unobscured radio-quiet AGN is analysed, these sources are among those with the lowest accretion rates (e.g., [113]). It is interesting to note that, although lacking the BLR, these objects do appear to have a face-on Compton-thick torus, as type 1 sources, as evidenced by the presence of silicate emission features in their IR spectra [114], and of a neutral iron emission line [109].

The existence of a critical threshold in luminosity and Eddington rate has been confirmed on observational grounds (e.g., [113, 115-118]). Below this threshold (whose exact value depends on the adopted sample, and the methods to derive the bolometric luminosities), no broad lines are detected (either in total or polarized light). On the other hand, it is clear that above the threshold the BLR still cannot be detected in many sources (see Figure 6). If the scenario proposed by Nicastro [106] is correct, these sources should possess a BLR, so there must be something that prevents us from observing it. Indeed, all these sources are Compton-thick, so the nucleus is severely obscured by intervening absorbers. It was suggested that this could be explained within the framework of standard Unification Models, whereby more inclined sources (with respect to the line of sight) should intercept a larger column density of the torus and may obscure the medium responsible for the scattering of the BLR photons (e.g., [117, and references therein]).

3.2. Disk-Torus Alignment. Although not explicitly required by any Unification Model, the most natural assumption on the geometry of the circumnuclear matter in AGN is that it is coaxial with the spin of the BH. This expectation is based on an angular conservation argument: if the obscuring 


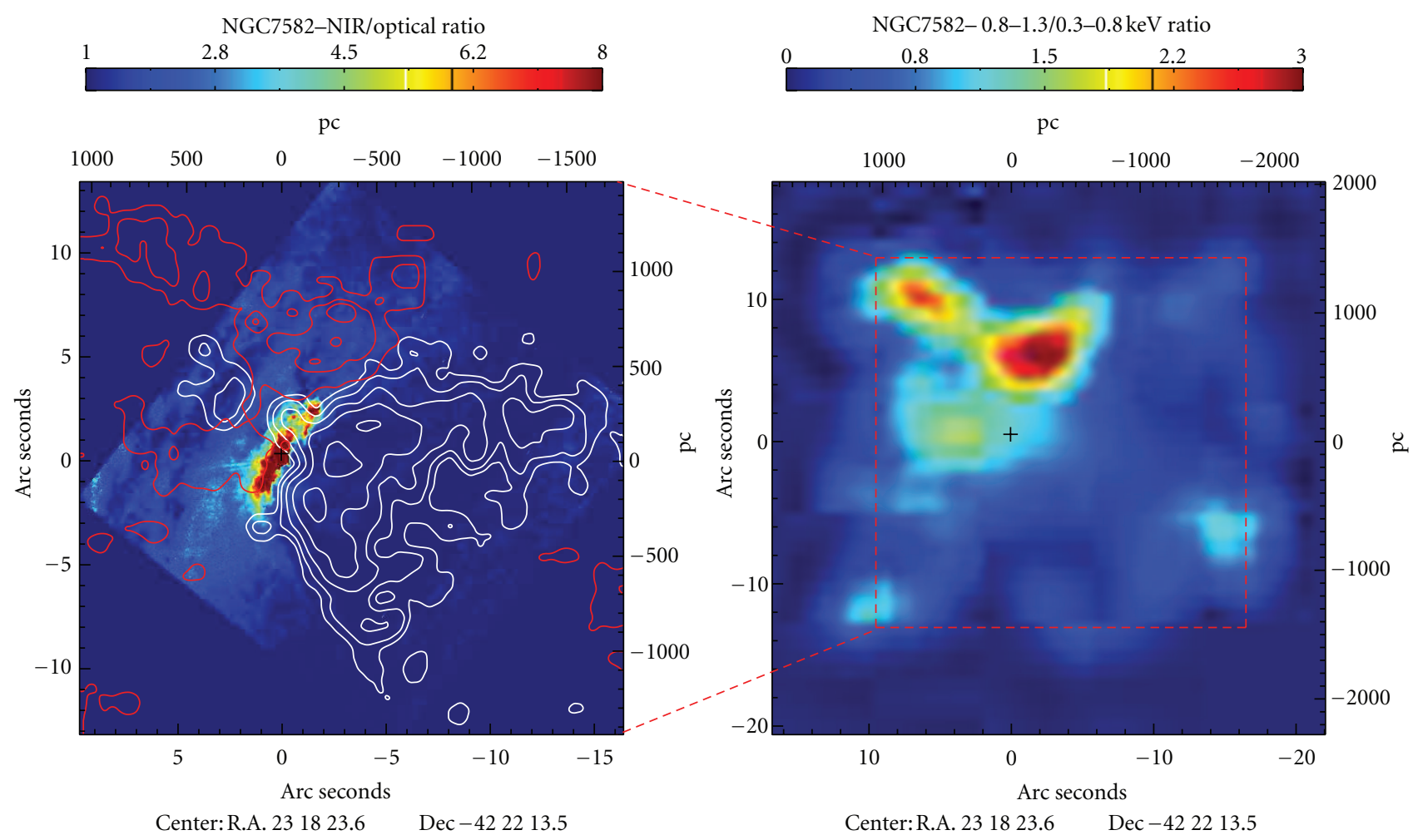

Figure 5: The Seyfert 2 galaxy NGC 7582 (from Bianchi et al. [95]). (a) HST NIR to optical ratio, mapping the amount of dust in the circumnuclear region of NGC 7582. The white contour plots refer to the Chandra emission below $0.8 \mathrm{keV}$, while the red ones the ratio shown in the right panel. (b) Chandra $0.8-1.3$ to $0.3-0.8 \mathrm{keV}$ ratio. The red contour plots shown in left panel refer to this image, but the scale is different, as outlined by the broken-line rectangle. In both panels, the black cross indicates the position of the nucleus.

torus is related to the inflowing material, it is natural to expect that the torus, the accretion disk, and the black hole rotation (mostly due to the angular momentum of the accreting material) share the same axis. This hypothesis, though reasonable, may not be verified in several more complex scenarios. For example, if the $\mathrm{BH}$ growth is due to multiple, unrelated accretion events, the actual $\mathrm{BH}$ spin may not reflect the rotation axis of the accretion disk. Another possibility is that the obscuring torus is not within the gravitational sphere of influence of the $\mathrm{BH}$ (e.g., a galactic dust lane). In this case, no obvious physical relation is expected between the torus axis and the $\mathrm{BH}$ spin. If the torus- $\mathrm{BH}$ spin alignment hypothesis is accepted, it implies that the accretion disk is aligned with the obscuring torus. Any radio jet should be aligned to the same axis. Finally, the orientation and the opening angle of the NLR ionization cones are collimated by the inner aperture of the torus, thus being themselves coaligned with the common disk/torus axis. These expectations are very difficult to test, due to the very small scales of the inner regions of AGN. When larger scales are imaged, at least the radio jet and the optical/X-ray NLR appear to be generally in agreement with this simple picture (e.g., $[119,120])$.

The mid-infrared interferometric studies described in Section 2.2 allowed us for the first time to directly image the geometry of the torus with respect to the optical cones.
Surprisingly, the results obtained by Raban et al. [70] strongly suggest that the two structures are misaligned in NGC 1068 (Figure 7). Moreover, the direction of the radio jet is also clearly tilted with respect to both the NLR and the torus. Some of the discrepancy can be solved by taking into account the detailed kinematics of the outflow, and by assuming a clumpy torus that could prevent the ionization of all the gas present in the geometrical opening angle of the ionization cones. However, these solutions are not in agreement with the results inferred from the appearance of the cones in the infrared [70, and references therein]. Similar analysis on other sources is clearly needed in order to shade some light on this issue. A promising, independent, method to test the torus/ionization cones misalignment is via Xray polarimetry, but we will have to wait for a future X-ray mission equipped with a broadband polarimeter [121].

It is more difficult to estimate the inclination angle of the accretion disk. One possible method is via the relativistic profile of iron emission lines produced in the inner regions of the disk, which is expected to be strongly dependent on the inclination angle (e.g., [122]). When sizeable samples of AGN are systematically analysed, it appears that a simple relation between the inclination of the nuclear obscuring matter (as measured by the optical type) and that of the accreting matter should be ruled out, in contrast with the naive expectations from Unified Models [123]. However, as 


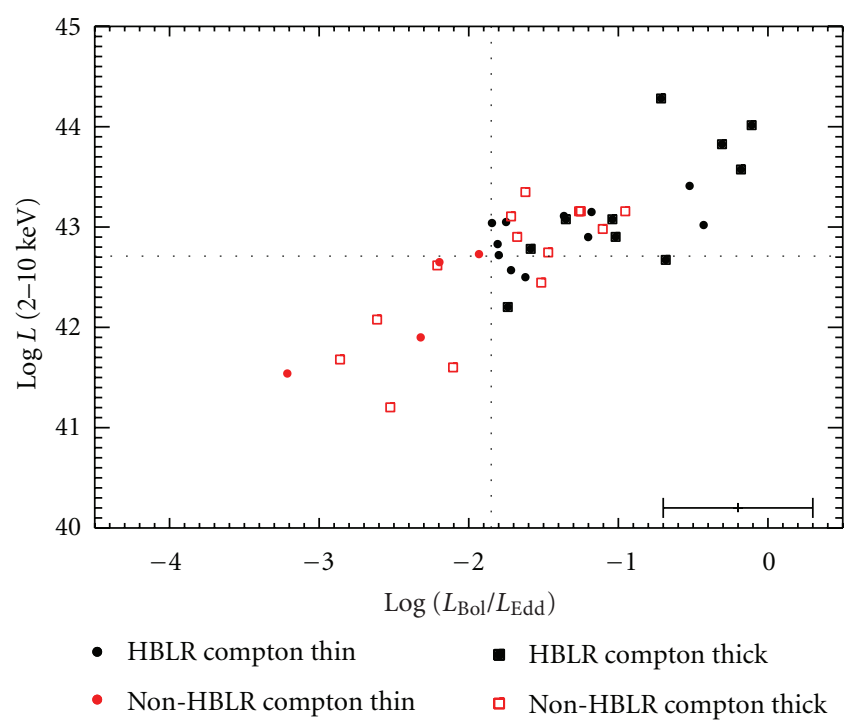

Figure 6: The separation between Seyfert 2 with detection of polarized broad lines (hidden BLR, HBLR) and Seyfert $2 \mathrm{~s}$ without polarized broad lines (non-HBLR) is clear both in X-ray luminosity and Eddington rate, when only Compton-thin sources are taken into account. However, a number of Compton-thick Non-HBLR sources have high accretion rates and luminosity, suggesting that obscuration also plays an important role in the detection of the HBLR (from Marinucci et al. [113]).

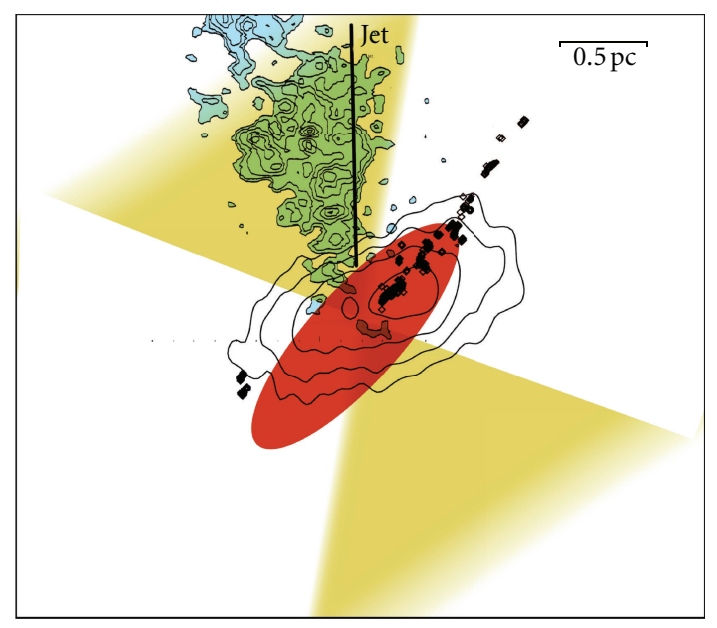

FIGURE 7: Sketch of the geometry of the circumnuclear matter in NGC 1068: compact dust inferred from mid-infrared interferometry (red), ionization cones as mapped by the [O III] emission (blue, reduced in scale by a factor $\sim 100$ ) and as inferred from spectroscopy (yellow). From Raban et al. [70].

noted by the same authors, the inclination angles derived from the profile of a relativistically broadened line are still affected by large systematic uncertainties. Another method was recently suggested by Risaliti et al. [124], who analysed the distribution of the equivalent widths of the [O III] emission line in a large sample of AGN. Their results are again not compatible with the presence of a torus coaligned with the accretion disk, unless the torus covering factor is extremely small.

Finally, evidence of a misalignment between disk and radio jet for a few individual sources comes from the analysis of the jet-disk relation in objects where the disk inclination can be inferred from maser emission. A prominent example of this kind of studies is that of NGC 3079 [67].

\subsection{Luminosity and Redshift Dependence of the Covering} Factor. An interesting finding of the recent years has been the evidence for a dependence of the obscuring medium covering factor on the AGN luminosity. More specifically, the covering factor of the obscuring medium appears to decrease significantly with luminosity. This effect has been shown quite clearly by various hard X-ray studies [125130] and by optical surveys [131], which have measured the relative fraction of obscured and unobscured AGN as a function of the bolometric luminosity. Clearly these works are affected by various uncertainties and caveats, primarily related to possible incompleteness effects and biases that may prevent the identification of obscured AGN in more distant galaxies (hence more luminous, as a consequence of the Malmquist bias). Indeed, these results have been questioned by some authors [132-134]. However, more recent extensive hard X-ray surveys have further confirmed a clear trend for a decreasing fraction of obscured AGN at high luminosity [135-138]. Figure 8 shows the results of some of these studies [135], where black and blue solid symbols show the fraction of obscured AGN as a function of X-ray luminosity, while red-dotted symbols illustrate the same trend from optical surveys [131].

The luminosity dependence of the covering factor has been recently questioned by Lawrence and Elvis [36], showing that, at least in optical and IR-selected sample, the luminosity dependence is largely a consequence of the inclusion of low excitation AGN (which may have an intrinsically different engine) and on the definition of "obscured" AGN (i.e., whether including mildly obscured AGN or not). However, the luminosity dependence of the covering factor still persists in the X-ray-selected samples, regardless of the classification scheme.

An alternative method to investigate the covering factor of the obscuring medium is by means of the dust reprocessing by the obscuring medium. More specifically, the ratio between the hot dust emission observed in the near/mid-IR and the primary AGN bolometric emission (optical/UV/Xray), responsible for heating the dust, is proportional to the covering factor of the obscuring medium. This method can only be applied to type $1 \mathrm{AGN}$, where the primary optical/UV radiation is directly detectable. By using ISO and Spitzer data to trace the dust emission, various studies have confirmed that the covering factor of the absorbing medium decreases as a function of luminosity, as shown in Figure 8 [62, 139, 141, 142] (but see also [143]). These IR results obtain a covering factor significantly higher than $\mathrm{X}$-ray studies, but this is likely due to the population of Compton-thick AGN that is mostly lost by X-ray surveys. 


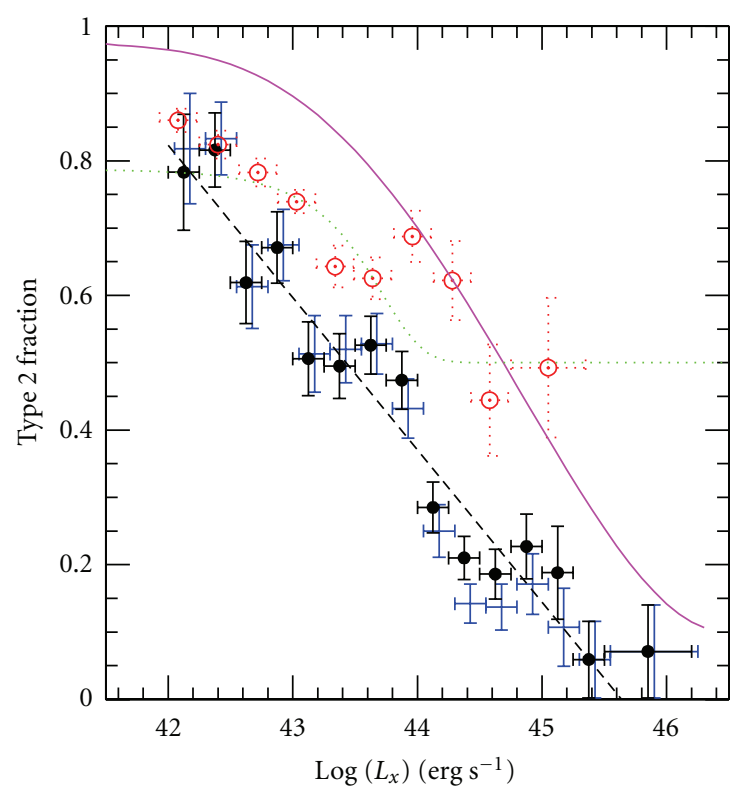

FIGURE 8: Fraction of type 2 AGN as a function of X-ray luminosity (from Hasinger [135]). The solid symbols show hard X-ray-selected AGN (blue and black symbols illustrate different subsamples in terms of redshift completeness). The red-dotted symbols show optically selected AGN (from Simpson [131]). The magenta line shows the type 2 fraction inferred from the dust covering factor obtained through near/mid-IR observations (from Maiolino et al. [139], note that these include also Compton-thick AGN, which are not present in X-ray samples). The green-dotted line shows the expected fraction of Compton-thin type 2 AGN according to the $\mathrm{X}$-ray background synthesis models given in Gilli et al. [140].

In a similar manner, the equivalent width of the (narrow) $\mathrm{Fe} \mathrm{K} \alpha$ line has been used as a proxy of the covering factor of the circumnuclear material responsible for producing the $\mathrm{Fe} \mathrm{K} \alpha$ line relative to the primary $\mathrm{X}$-ray continuum. The EW of the $\mathrm{Fe} \mathrm{K} \alpha$ has also been found to anticorrelate with luminosity ("X-ray Baldwin effect" or "Iwasawa-Taniguchi effect"), a trend which was generally interpreted in terms of decreasing covering factor of the circumnuclear absorbing medium as a function of luminosity [144-148].

Therefore, although partly questioned, several independent observational studies strongly favor a luminosity dependence of the covering factor of the circumnuclear absorbing medium. The origin of the anticorrelation between luminosity and covering factor is unclear. The "receding torus" scenario [149] is often invoked to explain this trend: higher luminosities imply a larger dust sublimation radius and, if the torus has a constant height as a function of radius, this results into a smaller covering factor of the dusty medium. However, this scenario cannot explain the results on the decreasing covering factor inferred from X-ray studies, which do not trace the dusty component of the absorber.

In the model proposed by Lamastra [150], the covering factor-luminosity anticorrelation naturally arises if the X-ray obscuration in Compton-thin sources is due to interstellar gas, distributed in a rotationally supported disk with an extension of a few hundred pc. The covering factor of this disc diminishes as the gravitational pull from the central $\mathrm{SMBH}$ and the bulge increases with the $\mathrm{BH}$ mass (and, therefore, the luminosity), producing the observed anticorrelation. However, this model can explain the anticorrelation only for Compton-thin sources.

Another possible scenario is that the lower covering factor in luminous AGN (quasars) is simply a consequence of the stronger AGN radiation pressure impinging onto the circumnuclear medium and expelling larger fractions of material. In support of this scenario growing evidence for massive outflows in luminous AGN has been reported in the recent years [151-155].

Some studies have also claimed an evolution of the covering factor with redshift. More specifically, by using large samples of X-ray selected objects, La Franca et al. [127] and Hasinger [135] have found evidence for a strong increase of the fraction of obscured AGN from $z=0$ to $z=$ 2. This result is however more debated, due to the larger uncertainties in disentangling luminosity and redshift effects in flux-limited samples and also more critically subject to selection effects in high- $z$ objects. Indeed, other authors did not find significant redshift dependence $[125,140]$, while Ballantyne et al. [156] and Treister and Urry [157] found a shallow redshift dependence of the fraction of obscured AGN. Here we only mention that an increase of the fraction of obscured AGN at high redshift is naturally expected by the larger gas content found in high- $z$ galaxies $[158,159]$ and associated with the enhanced star formation rates in high- $z$ galaxies, also observed in high- $z$ AGN host galaxies $[160,161]$.

A possible additional complication to the simple version of the Unified Model is that the covering factor of the obscuring medium may not only depend on the AGN luminosity, and possibly on redshift, but may also be intrinsically different between type 2 and type 1 AGN. Ricci et al. [162] have shown that the X-ray cold reflection "hump" in type $2 \mathrm{AGN}$ is stronger than in type $1 \mathrm{AGN}$, suggesting a larger covering factor of the circumnuclear medium in the former class. This result is, however, at odds with those obtained by mid-IR interferometry studies, as discussed above. More data are certainly required to tackle the discrepancy.

3.4. Unusual Geometries. While the general picture discussed in this paper can account for most of the observational properties observed in AGN, some subsamples require different geometries. This is the case for a number of nuclei that are unambiguously hosting a relatively powerful AGN, based on their X-ray or mid-IR properties, but which do not show any evidence for classical NLR tracers in their optical spectra [163-173]. A possibility is that in these objects the NLR, although extended, is heavily obscured by absorbing medium distributed on large scales in the host galaxy. Indeed, in a number of objects Spitzer spectroscopic observations have revealed mid-IR high excitation lines (e.g., [NeV] $14.3 \mu \mathrm{m})$ typical of the NLR [174-176].

However, a number of active nuclei do not show NLR-like lines even in the mid-IR [177]. In these cases a likely scenario 
is that the nuclear engine is obscured in all directions $(4 \pi$ obscuration) on small $(\sim \mathrm{pc})$ scales, so that UV photons cannot escape to produce an NLR. Further observational indications in favour of $4 \pi$ obscuration is the very small amount of reflection component at energies below $10 \mathrm{keV}$ in some Compton thick AGN e.g. [178, 179]. The most likely explanation in this case is that the Compton thick absorbing medium totally covers the nuclear sources, including the Compton reflecting medium.

If totally "buried" AGN may be one extreme of the population, on the other extreme there is growing evidence for a population of (type 1) AGN missing the circumnuclear absorber. Jiang et al. [180] discovered two quasars at $z \sim$ 6 showing no indication of hot dust emission typically observed in AGN. They interpreted this result as evidence for young quasars that had no time yet to form dust at such early epochs. However, evidence for AGN with little or no circumnuclear hot dust emission has been found also at lower redshifts $[142,181,182]$, in evolved quasars, where time scale for dust formation is likely not an issue. The nature of this class of objects is still unclear. Possible scenarios invoke dust destruction (dynamically or by radiation) or the AGN is not centered onto the supermassive black hole, as it may happen in the case of $\mathrm{BH}$ recoils in merging events, which may result in the off-nuclear AGN to be still surrounded by an accretion disk and a BLR, but not from pc-scale dusty medium [182].

\section{Summary and Conclusions}

The Unified Model for AGN has been tested in many different ways in the past few years, through a large set of new imaging, spectral, and timing observations. Overall, the fundamental aspect of the model, that is, that nonspherically symmetric absorption plays a major role in explaining the differences in the observed features among AGN, has been confirmed, and even reinforced by the most recent observations.

However, some more complexity has been added. In particular, the existence of the standard "torus", in the generic sense of an axisymmetric, rather than spherically symmetric, circumnuclear absorber has been confirmed, but its physical and geometrical structure has been proven to be far from homogeneous among the AGN family. There is now strong evidence of at least three absorption components on very different scales:

(i) on scales of hundreds of parsecs, or even larger (e.g., galactic dust lanes), circumnuclear tori have been imaged, with different techniques, and are clearly responsible of the "type 2" (in optical/UV) or "absorbed" (in X-rays) classification of a significant fraction of AGN;

(ii) on the parsec scale, and down to the dust sublimation radius, the "standard" torus, as initially postulated in the earliest works on AGN unification, has been now directly imaged in a few sources with interferometric techniques, and its presence is suggested by $\mathrm{X}$ ray reflection properties, and by dust reverberation mapping in the near-IR;

(iii) on the $0.01 \mathrm{pc}$ scale, the presence of dust-free gas along the line of sight has been demonstrated through X-ray absorption variability in several AGN, thus suggesting that part of the observed X-ray absorption is due to Broad Line Region clouds.

From the latter study, and from models of dust reemission in the infrared, it is also clear that the absorber has a clumpy, rather than homogeneous, structure.

Several "open issues" have been also briefly discussed in this paper, showing that not all AGN fit in a simple unification scheme, and that even within the general boundaries of the model, more work and new observations are needed to fully understand the dependence of all the observed properties on the physical parameters of the central source and of its environment.

\section{Acknowledgments}

The authors would like to thank the anonymous referees for useful suggestions, and R. Antonucci, M. Elvis, G. Matt, K. Tristram for feedback on the first version of the paper.

\section{References}

[1] R. Antonucci, "Unified models for active galactic nuclei and quasars," Annual Review of Astronomy and Astrophysics, vol. 31, no. 1, pp. 473-521, 1993.

[2] R. R. J. Antonucci, "Optical spectropolarimetry of radio galaxies," The Astrophysical Journal, vol. 278, pp. 499-520, 1984.

[3] C. K. Seyfert, "Nuclear emission in spiral nebulae," The Astrophysical Journal, vol. 97, p. 28, 1943.

[4] R. R. J. Antonucci and J. S.. Miller, "Diffuse pulmonary hemorrhage: a review and classification," The Astrophysical Journal, vol. 297, pp. 621-632, 1985.

[5] J. H. Krolik and M. C. Begelman, "An X-ray heated wind in NGC 1068," The Astrophysical Journal, vol. 308, pp. L55-L58, 1986.

[6] J. H. Krolik and M. C. Begelman, "Molecular tori in seyfert galaxies-feeding the monster and hiding it," The Astrophysical Journal, vol. 329, pp. 702-711, 1988.

[7] S. Kaspi, D. Maoz, H. Netzer, B. M. Peterson, M. Vestergaard, and B. T. Jannuzi, "The relationship between luminosity and broad-line region size in active galactic nuclei," The Astrophysical Journal, vol. 629, no. 1, pp. 61-71, 2005.

[8] B. M. Peterson, "Reverberation mapping of active galactic nuclei," Publications of the Astronomical Society of the Pacific, vol. 105, pp. 247-268, 1993.

[9] H. D. Tran, "Hidden broad-line Seyfert 2 galaxies in the CfA and $12 \mu \mathrm{m}$ samples," Astrophysical Journal Letters, vol. 554, no. 1, pp. L19-L23, 2001.

[10] S. Veilleux, R.-W. Goodrich, and G. J. Hill, "Infrared spectroscopy of seyfert 2 galaxies: a look through the obscuring torus? II," The Astrophysical Journal, vol. 477, article 631, 1997.

[11] S. Veilleux, R.-W. Goodrich, and G. J. Hill, "Infrared spectroscopy of seyfert 2 galaxies: a look through the obscuring 
torus? II," The Astrophysical Journal, vol. 477, no. 2, pp. 631660, 1997.

[12] N. M. Nagar, E. Oliva, A. Marconi, and R. Maiolino, "NGC 5506 unmasked as a narrow line Seyfert 1: a direct view of the broad line region using near-IR spectroscopy," Astronomy and Astrophysics, vol. 391, no. 2, pp. L21-L24, 2002.

[13] D. Lutz, R. Maiolino, A. F. M. Moorwood et al., "Infrared spectroscopy around 4 mu m of Seyfert 2 galaxies: Obscured broad line regions and coronal lines," Astronomy and Astrophysics, vol. 396, pp. 439-448, 2002.

[14] R. Riffel, A. Rodríguez-Ardila, and M. G. Pastoriza, "A 0.8$2.4 \mu \mathrm{m}$ spectral atlas of active galactic nuclei," Astronomy and Astrophysics, vol. 457, no. 1, pp. 61-70, 2006.

[15] H.-B. Cai, X.-W. Shu, Z.-Y. Zheng, and J. X. Wang, "The study of Seyfert 2 galaxies with and without infrared broad lines," Research in Astronomy and Astrophysics, vol. 10, no. 5, pp. 427-437, 2010.

[16] D. A. Smith and C. Done, "Unified theories of active galactic nuclei: a hard X-ray sample of Seyfert 2 galaxies," Monthly Notices of the Royal Astronomical Society, vol. 280, no. 2, pp. 355-377, 1996.

[17] T. J. Turner, I. M. George, K. Nandra, and R. F. Mushotzky, "ASCA observations of type 2 seyfert galaxies. I. Data analysis results," The Astrophysical Journal Supplement Series, vol. 113, no. 1, article 23, 1997.

[18] R. Maiolino, M. Salvati, L. Bassani et al., "Heavy obscuration in X-ray weak AGNs," Astronomy and Astrophysics, vol. 338, no. 3, pp. 781-794, 1998.

[19] L. Bassani, M. Dadina, R. Maiolino et al., "A threedimensional diagnostic diagram for Seyfert 2 galaxies: probing X-ray absorption and compton thickness," Astrophysical Journal, Supplement Series, vol. 121, no. 2, pp. 473-482, 1999.

[20] G. Matt, M. Guainazzi, F. Frontera et al., "Hard X-ray detection of NGC 1068 with BeppoSAX," Astronomy and Astrophysics, vol. 325, no. 2, pp. L13-L16, 1997.

[21] G. Matt, M. Guainazzi, R. Maiolino et al., "One more surprise from the circinus galaxy: beppoSAX discovery of a transmission component in hard X-rays," Astronomy and Astrophysics, vol. 341, no. 2, pp. L39-L42, 1999.

[22] R. W. Pogge, "Extended ionized gas in the Seyfert 2 galaxy NGC 4388," The Astrophysical Journal, vol. 332, pp. 702-710, 1988.

[23] I. N. Evans, H. C. Ford, A. L. Kinney, R. R. J. Antonucci, L. Armus, and S. Caganoff, "HST imaging of the inner 3 arcseconds of NGC 1068 in the light of [O III] ג5007," The Astrophysical Journal, vol. 369, no. 2, pp. L27-L30, 1991.

[24] A. S. Wilson and Z. I. Tsvetanov, "Ionization cones and radio ejecta in active galaxies," Astronomical Journal, vol. 107, no. 4, pp. 1227-1234, 1994.

[25] F. K. B. Barbosa, T. Storchi-Bergmann, R. C. Fernandes, C. Winge, and H. Schmitt, "Gemini/GMOS IFU gas velocity 'tomography' of the narrow line region of nearby active galaxies," Monthly Notices of the Royal Astronomical Society, vol. 396, no. 1, pp. 2-18, 2009.

[26] R. Maiolino and G. H. Rieke, "Low-luminosity and obscured seyfert nuclei in nearby galaxies," The Astrophysical Journal, vol. 454, no. 1, pp. 95-105, 1995.

[27] N. M. Nagar, A. S. Wilson, J. S. Mulchaey, and J. F. Gallimore, "Radio structures of Seyfert galaxies. VIII. A distance- and magnitude-limited sample of early-type galaxies," Astrophysical Journal, Supplement Series, vol. 120, no. 2, pp. 209-245, 1999.
[28] E. A. Pier and J. H. Krolik, "Radiation-pressure-supported obscuring tori around active galactic nuclei," The Astrophysical Journal, vol. 399, no. 1, pp. L23-L26, 1992.

[29] G. L. Granato, L. Danese, and A. Franceschini, "Thick tori around active galactic nuclei: the case for extended tori and consequences for their X-ray and infrared emission," The Astrophysical Journal, vol. 486, no. 1, pp. 147-159, 1997.

[30] J. H. Krolik, "AGN obscuring tori supported by infrared radiation pressure," The Astrophysical Journal, vol. 661, no. 1, pp. 52-59, 2007.

[31] K. Wada and K. Tomisaka, "Molecular gas structure around an active galactic nucleus with nuclear starburst: threedimensional non-LTE calculations of CO lines," The Astrophysical Journal, vol. 619, no. 1 I, pp. 93-104, 2005.

[32] Y. Watabe and M. Umemura, "Obscuration of active galactic nuclei by circumnuclear starbursts," The Astrophysical Journal, vol. 618, no. 2 I, pp. 649-656, 2005.

[33] S. Nayakshin, "Warped accretion discs and the unification of active galactic nuclei," Monthly Notices of the Royal Astronomical Society, vol. 359, no. 2, pp. 545-550, 2005.

[34] A. Caproni, Z. Abraham, and H. J. Mosquera Cuesta, "Bardeen-petterson effect and the disk structure of the seyfert galaxy NGC 1068," The Astrophysical Journal, vol. 638, no. 1 I, pp. 120-124, 2006.

[35] A. Caproni, M. Livio, Z. Abraham, and H. J. M. Cuesta, "Warping and precession in galactic and extragalactic accretion disks," The Astrophysical Journal, vol. 653, no. 1 I, pp. 112-126, 2006.

[36] A. Lawrence and M. Elvis, "Misaligned disks as obscurers in active galaxies," The Astrophysical Journal, vol. 714, no. 1, pp. 561-570, 2010.

[37] M. Elitzur and I. Shlosman, "The AGN-obscuring torus: the end of the "doughnut" paradigm?" The Astrophysical Journal, vol. 648, no. 2, pp. L101-L104, 2006.

[38] M. Nenkova, M. M. Sirocky, R. Nikutta, Z. Ivezi, and M. Elitzur, "AGN dusty Tori. II. observational implications of clumpiness," The Astrophysical Journal, vol. 685, no. 1, pp. 160-180, 2008.

[39] S. F. Hönig and T. Beckert, "Active galactic nuclei dust tori at low and high luminosities," Monthly Notices of the Royal Astronomical Society, vol. 380, no. 3, pp. 1172-1176, 2007.

[40] S. F. Hönig and M. Kishimoto, "The dusty heart of nearby active galaxies: II. from clumpy torus models to physical properties of dust around AGN," Astronomy and Astrophysics, vol. 523, no. 2, article no. A27, 2010.

[41] G. Risaliti, "The BeppoSAX view of bright compton-thin Seyfert 2 galaxies," Astronomy and Astrophysics, vol. 386, no. 2, pp. 379-398, 2002.

[42] G. Risaliti, M. Elvis, G. Fabbiano, A. Baldi, and A. Zezas, "Rapid compton-thick/compton-thin transitions in the seyfert 2 galaxy NGC 1365," The Astrophysical Journal, vol. 623, no. 2, pp. L93-L96, 2005.

[43] G. Risaliti, M. Elvis, G. Fabbiano, A. Baldi, A. Zezas, and M. Salvati, "Occultation measurement of the size of the X-rayemitting region in the active galactic nucleus of NGC 1365," The Astrophysical Journal, vol. 659, no. 2, pp. L111-L114, 2007.

[44] G. Risaliti, M. Salvati, M. Elvis et al., "The XMM-Newton long look of NGC 1365: uncovering of the obscured X-ray source," Monthly Notices of the Royal Astronomical Society, vol. 393, no. 1, pp. L1-L5, 2009.

[45] R. Maiolino, G. Risaliti, M. Salvati et al., "Comets orbiting a black hole," Astronomy and Astrophysics, vol. 517, no. 6, article A47, 2010. 
[46] M. Elvis, G. Risaliti, F. Nicastro, J. M. Miller, F. Fiore, and S. Puccetti, "An unveiling event in the type 2 active galactic nucleus NGC 4388: a challenge for a parsec-scale absorber," The Astrophysical Journal, vol. 615, no. 1, pp. L25-L28, 2004.

[47] S. Puccetti, F. Fiore, G. Risaliti, M. Capalbi, M. Elvis, and F. Nicastro, "Rapid NH changes in NGC 4151," Monthly Notices of the Royal Astronomical Society, vol. 377, no. 2, pp. 607-616, 2007.

[48] S. Bianchi, E. Piconcelli, M. Chiaberge, E. J. Bailon, G. Matt, and F. Fiore, "How complex is the obscuration in active galactic nuclei? New clues from the suzaku monitoring of the X-ray absorbers in NGC 7582," The Astrophysical Journal, vol. 695 , no. 1, pp. 781-787, 2009.

[49] G. Risaliti, E. Nardini, M. Salvati et al., "X-ray absorption by broad-line region clouds in Mrk 766," Monthly Notices of the Royal Astronomical Society, vol. 410, no. 2, pp. 1027-1035, 2011.

[50] R. Maiolino, A. Marconi, M. Salvati et al., "Dust in active nuclei I. Evidence for "anomalous" properties," Astronomy and Astrophysics, vol. 365, no. 2, pp. 28-36, 2001.

[51] T. Maccacaro, G. C. Perola, and M. Elvis, "X-ray observations of emission line galaxies with the einstein observatory," Space Science Reviews, vol. 30, no. 1-4, pp. 61-65, 1981.

[52] T. Maccacaro, G. C. Perola, and M. Elvis, "X-ray observations with the Einstein Observatory of emission-line galaxies," The Astrophysical Journal, vol. 257, p. 47, 1982.

[53] S. Bianchi, G. Matt, I. Balestra, and G. C. Perola, "The origin of the iron lines in NGC 7213," Astronomy and Astrophysics, vol. 407, no. 2, pp. L21-L24, 2003.

[54] S. Bianchi, G. Matt, I. Balestra, M. Guainazzi, and G. C. Perola, "X-ray reprocessing in Seyfert galaxies: simultaneous XMM-Newton/BeppoSAX observations," Astronomy and Astrophysics, vol. 422, no. 1, pp. 65-76, 2004.

[55] A. P. Lobban, J. N. Reeves, D. Porquet et al., "Evidence for a truncated accretion disc in the low-luminosity Seyfert galaxy, NGC 7213?" Monthly Notices of the Royal Astronomical Society, vol. 408, no. 1, pp. 551-564, 2010.

[56] S. Bianchi, F. La Franca, G. Matt et al., "A broad-line region origin for the iron K $\alpha$ line in NGC 7213," Monthly Notices of the Royal Astronomical Society, vol. 389, no. 1, pp. L52-L56, 2008.

[57] T. Storchi-Bergmann, J. S. Mulchaey, and A. S. Wilson, "Infrared emission in Seyfert 2 galaxies: reprocessed radiation from a dusty torus?" The Astrophysical Journal, vol. 395, no. 2, pp. L73-L77, 1992.

[58] A. Alonso-Herrero, A. C. Quillen, C. Simpson, A. Efstathiou, and M. J. Ward, "The nonstellar infrared continuum of seyfert galaxies," Astronomical Journal, vol. 121, no. 3, pp. 1369-1384, 2001.

[59] E. Oliva, L. Origlia, R. Maiolino, and A. F. M. Moorwood, "Starbursts in active galaxy nuclei: observational constraints from IR stellar absorption lines," Astronomy and Astrophysics, vol. 350, no. 1, pp. 9-16, 1999.

[60] M. Suganuma, Y. Yoshii, Y. Kobayashi et al., "Reverberation measurements of the inner radius of the dust torus in nearby seyfert 1 galaxies," The Astrophysical Journal, vol. 639, no. 1, pp. 46-63, 2006.

[61] R. Maiolino, O. Shemmer, M. Imanishi et al., "Dust covering factor, silicate emission, and star formation in luminous QSOs," Astronomy and Astrophysics, vol. 468, no. 3, pp. 979992, 2007.

[62] E. Treister, J. H. Krolik, and C. Dullemond, "Measuring the fraction of obscured quasars by the infrared luminosity of unobscured quasars," The Astrophysical Journal, vol. 679, no. 1, pp. 140-148, 2008.

[63] L. J. Greenhill, C. R. Gwinn, R. Antonucci, and R. Barvainis, "VLBI imaging of water maser emission from the nuclear torus of NGC 1068," The Astrophysical Journal, vol. 472, no. 1, pp. L21-L24, 1996.

[64] L. J. Greenhill, J. M. Moran, and J. R. Herrnstein, "The distribution of $\mathrm{H}_{2} \mathrm{O}$ maser emission in the nucleus of NGC 4945," The Astrophysical Journal, vol. 481, no. 1, pp. L23-L26, 1997.

[65] L. J. Greenhill, P. T. Kondratko, J. E. J. Lovell et al., “The discovery of $\mathrm{H}_{2} \mathrm{O}$ maser emission in seven active galactic nuclei and at high velocities in the Circinus galaxy," The Astrophysical Journal, vol. 582, no. 1, pp. L11-L14, 2003.

[66] P. T. Kondratko, L. J. Greenhill, and J. M. Moran, "The parsec-scale accretion disk in NGC 3393," The Astrophysical Journal, vol. 678, no. 1, pp. 87-95, 2008.

[67] P. T. Kondratko, L. J. Greenhill, and J. M. Moran, "Evidence for a geometrically thick self-gravitating accretion disk in NGC 3079," The Astrophysical Journal, vol. 618, no. 2, pp. 618-634, 2005.

[68] J. F. Gallimore, S. A. Baum, and C. P. O’Dea, "A direct image of the obscuring disk surrounding an active galactic nucleus," Nature, vol. 388, no. 6645, pp. 852-854, 1997.

[69] W. Jaffe, K. Meisenheimer, H. J. A. Röttgering et al., "The central dusty torus in the active nucleus of NGC 1068," Nature, vol. 429, no. 6987, pp. 47-49, 2004.

[70] D. Raban, W. Jaffe, H. Röttgering, K. Meisenheimer, and K. R. W. Tristram, "Resolving the obscuring torus in NGC 1068 with the power of infrared interferometry: revealing the inner funnel of dust," Monthly Notices of the Royal Astronomical Society, vol. 394, no. 3, pp. 1325-1337, 2009.

[71] K. R. W. Tristram, K. Meisenheimer, W. Jaffe et al., "Resolving the complex structure of the dust torus in the active nucleus of the Circinus galaxy," Astronomy and Astrophysics, vol. 474, no. 3, pp. 837-850, 2007.

[72] L. Burtscher, W. Jaffe, D. Raban, K. Meisenheimer, K. R. W. Tristram, and H. Röttgering, "Dust emission from a parsecscale structure in the seyfert 1 nucleus of NGC 4151," The Astrophysical Journal, vol. 705, no. 1, pp. L53-L57, 2009.

[73] K. R. W. Tristram, D. Raban, K. Meisenheimer et al., "Parsecscale dust distributions in Seyfert galaxies Results of the MIDI AGN snapshot survey," Astronomy and Astrophysics, vol. 502, no. 1, pp. 67-84, 2009.

[74] K. R. W. Tristram and M. Schartmann, "On the sizeluminosity relation of AGN dust tori in the mid-infrared," Astronomy and Astrophysics, vol. 531, article A99, 2011.

[75] M. Kishimoto, S. F. Honig, R. Antonucci et al., "The innermost dusty structure in active galactic nuclei as probed by the Keck interferometer," Astronomy \& Astrophysics, vol. 527, article A121, 2011.

[76] G. C. Perola, G. Matt, M. Cappi et al., "Compton reflection and iron fluorescence in BeppoSAX observations of Seyfert type 1 galaxies," Astronomy and Astrophysics, vol. 389, no. 3, pp. 802-811, 2002.

[77] S. Bianchi, M. Guainazzi, G. Matt, N. Fonseca Bonilla, and G. Ponti, "CAIXA: a catalogue of AGN in the XMM-Newton archive: I. Spectral analysis," Astronomy and Astrophysics, vol. 495, no. 2, pp. 421-430, 2009.

[78] I. De La Calle Pérez, A. L. Longinotti, M. Guainazzi et al., "FERO: finding extreme relativistic objects: I. Statistics of relativistic $\mathrm{Fe} \mathrm{K} \alpha$ lines in radio-quiet Type 1 AGN," Astronomy and Astrophysics, vol. 524, no. 2, article no. A50, 2010 . 
[79] K. Nandra, P. M. O’Neill, I. M. George, and J. N. Reeves, "An XMM-Newton survey of broad iron lines in Seyfert galaxies," Monthly Notices of the Royal Astronomical Society, vol. 382, no. 1, pp. 194-228, 2007.

[80] K. Nandra, "On the origin of the iron Ko line cores in active galactic nuclei," Monthly Notices of the Royal Astronomical Society, vol. 368, pp. L62-L66, 2006.

[81] X. W. Shu, T. Yaqoob, and J. X. Wang, "Chandra High-energy Grating Observations of the $\mathrm{Fe} \mathrm{K} \alpha$ Line Core in Type II Seyfert Galaxies: A Comparison with Type I Nuclei," The Astrophysical Journal, vol. 738, p. 147, 2011.

[82] R. Gilli, R. Maiolino, A. Marconi et al., "The variability of the Seyfert galaxy NGC 2992: the case for a revived AGN," Astronomy and Astrophysics, vol. 355, no. 2, pp. 485-498, 2000.

[83] G. Matt, S. Bianchi, M. Guainazzi et al., "Chandra and XMMNewton observations of Tololo 0109-383," Astronomy and Astrophysics, vol. 399, no. 2, pp. 519-523, 2003.

[84] M. Nenkova, Z. Ivezi, and M. Elitzur, "Dust emission from active galactic nuclei," The Astrophysical Journal, vol. 570, no. 1, pp. L9-L12, 2002.

[85] M. Elitzur and I. Shlosman, "The AGN-obscuring torus: the end of the "doughnut" paradigm?" The Astrophysical Journal, vol. 648, no. 2, pp. L101-L104, 2006.

[86] S. F. Hönig, T. Beckert, K. Ohnaka, and G. Weigelt, "Radiative transfer modeling of three-dimensional clumpy AGN tori and its application to NGC 1068," Astronomy and Astrophysics, vol. 452, no. 2, pp. 459-471, 2006.

[87] G. L. Granato and L. Danese, "Thick tori around active galactic nuclei-a comparison of model predictions with observations of the infrared continuum and silicate features," Monthly Notices of the Royal Astronomical Society, vol. 268, no. 1, p. 235, 1994.

[88] R. Maiolino, "Prospects for AGN studies with ALMA," New Astronomy Reviews, vol. 52, no. 6, pp. 339-357, 2008.

[89] Y. Shi, G. H. Rieke, D. C. Hines et al., "9.7 $\mu \mathrm{m}$ silicate features in active galactic nuclei: new insights into unification models," The Astrophysical Journal, vol. 653, no. 1 I, pp. 127136, 2006.

[90] R. Nikutta, M. Elitzur, and M. Lacy, "On the $10 \mu \mathrm{m}$ silicate feature in active galactic nuclei," The Astrophysical Journal, vol. 707, no. 2, pp. 1550-1559, 2009.

[91] G. Matt, "Dust lanes, thick absorbers, and the unification model for Seyfert galaxies," Astronomy and Astrophysics, vol. 355, no. 2, pp. L31-L33, 2000.

[92] C. D. P. Lagos, N. D. Padilla, M. A. Strauss, S. A. Cora, and L. Hao, "Host galaxy-active galactic nucleus alignments in the sloan digital sky survey data release 7," Monthly Notices of the Royal Astronomical Society, vol. 414, no. 3, pp. 2148-2162, 2011.

[93] M. A. Malkan, V. Gorjian, and R. Tam, "A hubble space telescope 1 imaging survey of nearby active galactic nuclei," Astrophysical Journal, Supplement Series, vol. 117, no. 1, pp. 25-88, 1998.

[94] M. Guainazzi, G. Matt, and G. C. Perola, "X-ray obscuration and obscured AGN in the local universe," Astronomy and Astrophysics, vol. 444, no. 1, pp. 119-132, 2005.

[95] S. Bianchi, M. Chiaberge, E. Piconcelli, and M. Guainazzi, "A multiwavelength map of the nuclear region of NGC 7582," Monthly Notices of the Royal Astronomical Society, vol. 374, no. 2, pp. 697-702, 2007.

[96] E. Schinnerer, A. Eckart, L. J. Tacconi, R. Genzel, and D. Downes, "Bars and warps traced by the molecular gas in the
Seyfert 2 galaxy NGC 1068," The Astrophysical Journal, vol. 533, no. 2, pp. 850-868, 2000.

[97] F. Boone, S. García-Burillo, F. Combes et al., "Highresolution mapping of the physical conditions in two nearby active galaxies based on $12 \mathrm{CO}(1-0),(2-1)$, and (3-2) lines," Astronomy and Astrophysics, vol. 525, no. 1, article A18, 2010.

[98] M. Krips, S. Martin, and A. Eckart, "SMA/PdBI multiple line observations of the nearby Seyfert2 galaxy NGC 1068: shock related gas kinematics and heating in the central 100pc?" The Astrophysical Journal, vol. 736, article 37, 2011.

[99] M. Polletta, D. Weedman, S. Hönig, C. J. Lonsdale, H. E. Smith, and J. Houck, "Obscuration in extremely luminous quasars," The Astrophysical Journal, vol. 675, no. 2, pp. 960984, 2008.

[100] A. Martínez-Sansigre, S. Rawlings, M. Lacy et al., "A population of high-redshift type 2 quasars - I. Selection criteria and optical spectra," Monthly Notices of the Royal Astronomical Society, vol. 370, no. 3, pp. 1479-1498, 2006.

[101] G. Risaliti, R. Maiolino, and M. Salvati, "The distribution of absorbing column densities among Seyfert 2 galaxies," The Astrophysical Journal, vol. 522, no. 1, pp. 157-164, 1999.

[102] A. J. Young, A. S. Wilson, and P. L. Shopbell, "A Chandra X-ray study of NGC 1068. I. Observations of extended emission," The Astrophysical Journal, vol. 556, no. 1, pp. 623, 2001.

[103] C. A. Heisler, S. L. Lumsden, and J. A. Bailey, "Visibility of scattered broad-line emission in Seyfert 2 galaxies," Nature, vol. 385 , no. 6618 , pp. 700-702, 1997.

[104] D. M. Alexander, "The unified model and the Seyfert 2 infrared dichotomy," Monthly Notices of the Royal Astronomical Society, vol. 320, no. 1, pp. L15-L19, 2001.

[105] Q. Gu, R. Maiolino, and D. Dultzin-Hacyan, "Nuclear obscuration and scattering in Seyfert 2 galaxies," Astronomy and Astrophysics, vol. 366, no. 3, pp. 765-770, 2001.

[106] F. Nicastro, "Broad emission line regions in active galactic nuclei: the link with the accretion power," The Astrophysical Journal Letters, vol. 530, no. 2, article L65, 2000.

[107] M. Elitzur and L. C. Ho, "On the disappearance of the broadline region in low-luminosity active galactic nuclei," The Astrophysical Journal Letters, vol. 701, no. 2, article L91, 2009.

[108] J. R. Trump, C. D. Impey, B. C. Kelly et al., "Accretion rate and the physical nature of unobscured active galaxies," The Astrophysical Journal, vol. 733, no. 1, article 60, 2011.

[109] S. Bianchi, A. Corral, F. Panessa et al., "NGC 3147: a "true" type 2 Seyfert galaxy without the broad-line region," Monthly Notices of the Royal Astronomical Society, vol. 385, no. 1, pp. 195-199, 2008.

[110] F. Panessa, F. J. Carrera, S. Bianchi et al., "Unabsorbed Seyfert 2 galaxies: the case of "naked" AGN," Monthly Notices of the Royal Astronomical Society, vol. 398, no. 4, pp. 1951-1960, 2009.

[111] M. Brightman and K. Nandra, "On the nature of unabsorbed Seyfert 2 galaxies," Monthly Notices of the Royal Astronomical Society, vol. 390, no. 3, pp. 1241-1249, 2008.

[112] S. Bianchi, in prep., 2012.

[113] A. Marinucci, S. Bianchi, F. Nicastro, G. Matt, and A. D. Goulding, "The link between the hidden broad line region andthe accretion rate in seyfert 2 galaxies," The Astrophysical Journal. In press.

[114] Y. Shi, G. H. Rieke, P. Smith et al., "Unobscured type 2 active galactic nuclei," The Astrophysical Journal, vol. 714, no. 1, pp. 115-129, 2010.

[115] F. Nicastro, A. Martocchia, and G. Matt, "The lack of broadline regions in low accretion rate active galactic nuclei 
as evidence of their origin in the accretion disk," The Astrophysical Journal, vol. 589, no. 1, pp. L13-L16, 2003.

[116] W. Bian and Q. Gu, "The eddington ratios in seyfert 2 galaxies with and without hidden broad-line regions," The Astrophysical Journal, vol. 657, no. 1, pp. 159-166, 2007.

[117] X. W. Shu, J. X. Wang, P. Jiang, L. L. Fan, and T. G. Wang, "Investigating the nuclear obscuration in two types of Seyfert 2 galaxies," The Astrophysical Journal, vol. 657, no. 1 I, pp. 167-176, 2007.

[118] Y. Wu, E. Zhang, Y. Liang, C. Zhang, and Y. Zhao, "The different nature of seyfert 2 galaxies with and without hidden broad-line regions," The Astrophysical Journal, vol. 730, no. 2, p. 121, 2011.

[119] A. Capetti, D. J. Axon, F. Macchetto, W. B. Sparks, and A. Boksenberg, "Radio outflows and the origin of the narrowline region in seyfert galaxies," The Astrophysical Journal, vol. 469, no. 2, pp. 554-563, 1996.

[120] S. Bianchi, M. Chiaberge, D. A. Evans et al., "High-resolution X-ray spectroscopy and imaging of Mrk 573," Monthly Notices of the Royal Astronomical Society, vol. 405, no. 1, pp. 553-563, 2010.

[121] R. W. Goosmann and G. Matt, "Spotting the misaligned outflows in NGC 1068 using X-ray polarimetry," Monthly Notices of the Royal Astronomical Society, vol. 415, no. 4, pp. 3119-3128, 2011.

[122] A. C. Fabian, K. Iwasawa, C. S. Reynolds, and A. J. Young, "Broad iron lines in active galactic nuclei," Publications of the Astronomical Society of the Pacific, vol. 112, no. 775, pp. 11451161, 2000.

[123] M. Guainazzi, S. Bianchi, I. de La Calle Perez, M. Dovciak, and A. L. Longinotti, "On the driver of relativistic effect strength in Seyfert galaxies," Astronomy \& Astrophysics, vol. 531, article A131, 2011.

[124] G. Risaliti, M. Salvati, and A. Marconi, "[O iii] equivalent width and orientation effects in quasars," Monthly Notices of the Royal Astronomical Society, vol. 411, no. 4, pp. 2223-2229, 2011.

[125] Y. Ueda, M. Akiyama, K. Ohta, and T. Miyaji, "Cosmological evolution of the hard X-ray active galactic nucleus luminosity function and the origin of the hard X-ray background," The Astrophysical Journal, vol. 598, no. 2 I, pp. 886-908, 2003.

[126] A. T. Steffen, A. J. Barger, L. L. Cowie, R. F. Mushotzky, and Y. Yang, "The changing active galactic nucleus population," The Astrophysical Journal, vol. 596, no. 1, pp. L23-L26, 2003.

[127] F. La Franca, F. Fiore, A. Comastri et al., "The HELLAS2XMM survey. VII. The hard X-ray luminosity function of AGNs up to $\mathrm{z}=4$ : more absorbed AGNs at low luminosities and high redshifts," The Astrophysical Journal, vol. 635, no. 2 I, pp. 864-879, 2005.

[128] A. Akylas, I. Georgantopoulos, A. Georgakakis, S. Kitsionas, and E. Hatziminaoglou, "XMM-Newton and Chandra measurements of the AGN intrinsic absorption: dependence on luminosity and redshift," Astronomy and Astrophysics, vol. 459, no. 3, pp. 693-701, 2006.

[129] A. J. Barger and L. L. Cowie, "The number density of intermediate- and high-luminosity active galactic nuclei at $\mathrm{z} \sim 2-3$," The Astrophysical Journal, vol. 635, no. 1 I, pp. 115-122, 2005.

[130] P. Tozzi, R. Gilli, V. Mainieri et al., "X-ray spectral properties of active galactic nuclei in the Chandra Deep Field South," Astronomy and Astrophysics, vol. 451, no. 2, pp. 457-474, 2006.
[131] C. Simpson, "The luminosity dependence of the type 1 active galactic nucleus fraction," Monthly Notices of the Royal Astronomical Society, vol. 360, no. 2, pp. 565-572, 2005.

[132] T. Dwelly and M. J. Page, "The distribution of absorption in AGN detected in the XMM-Newton observations of the CDFS," Monthly Notices of the Royal Astronomical Society, vol. 372, no. 4, pp. 1755-1775, 2006.

[133] E. Treister and C. M. Urry, "Active galactic nuclei unification and the X-ray background," The Astrophysical Journal, vol. 630, no. 1 I, pp. 115-121, 2005.

[134] J.-M. Wang and E.-P. Zhang, "The unified model of active galactic nuclei. II. Evolutionary connection," The Astrophysical Journal, vol. 660, no. 2 I, pp. 1072-1092, 2007.

[135] G. Hasinger, "Absorption properties and evolution of active galactic nuclei," Astronomy and Astrophysics, vol. 490, no. 3, pp. 905-922, 2008.

[136] R. Della Ceca, A. Caccianiga, P. Severgnini et al., "The cosmological properties of AGN in the XMM-Newton Hard Bright Survey," Astronomy and Astrophysics, vol. 487, no. 1, pp. 119-130, 2008.

[137] E. Treister, S. Virani, E. Gawiser et al., "Optical spectroscopy of x-ray sources in the extended chandra deep field south," The Astrophysical Journal, vol. 693, no. 2, p. 1713, 2009.

[138] M. Brusa, F. Civano, A. Comastri et al., "The XMM-newton wide-field survey in the cosmos field (XMM-COSMOS): demography and multiwavelength properties of obscured and unobscured luminous active galactic nuclei," The Astrophysical Journal, vol. 716, no. 1, pp. 348-369, 2010.

[139] R. Maiolino, O. Shemmer, M. Imanishi et al., "Dust covering factor, silicate emission, and star formation in luminous QSOs," Astronomy and Astrophysics, vol. 468, no. 3, pp. 979992, 2007.

[140] R. Gilli, A. Comastri, and G. Hasinger, "The synthesis of the cosmic X-ray background in the Chandra and XMM-Newton era," Astronomy and Astrophysics, vol. 463, no. 1, pp. 79-96, 2007.

[141] J.-M. Wang, E.-P. Zhang, and B. Luo, "Evolutionary consequences of dusty tori in active galactic nuclei," The Astrophysical Journal, vol. 627, no. 1, article L5, 2005.

[142] R. Mor and B. Trakhtenbrot, "Hot-dust clouds with puregraphite composition around type-i active galactic nuclei," The Astrophysical Journal Letters, vol. 737, no. 2, p. L36, 2011.

[143] M. Rowan-Robinson, I. Valtchanov, and K. Nandra, "AGN dust tori: the X-ray-infrared connection," Monthly Notices of the Royal Astronomical Society, vol. 397, no. 3, pp. 1326-1337, 2009.

[144] K. Iwasawa and Y. Taniguchi, "The X-ray baldwin effect," The Astrophysical Journal, vol. 413, no. 1, pp. L15-L18, 1993.

[145] K. L. Page, P. T. O’Brien, J. N. Reeves, and M. J. L. Turner, "An X-ray Baldwin effect for the narrow Fe K $\alpha$ lines observed in active galactic nuclei," Monthly Notices of the Royal Astronomical Society, vol. 347, no. 1, pp. 316-322, 2004.

[146] P. Jiang, J. X. Wang, and T. G. Wang, "On the X-ray Baldwin effect for narrow Fe Ka emission lines," The Astrophysical Journal, vol. 644, no. 2, pp. 725-732, 2006.

[147] M. Guainazzi, S. Bianchi, and M. Dovčiak, "Statistics of relativistically broadened Fe K $\alpha$ lines in AGN," Astronomische Nachrichten, vol. 327, no. 10, pp. 1032-1038, 2006.

[148] S. Bianchi, M. Guainazzi, G. Matt, and N. F. Bonilla, "On the Iwasawa-Taniguchi effect of radio-quiet AGN," Astronomy and Astrophysics, vol. 467, no. 1, pp. L19-L22, 2007.

[149] A. Lawrence, "The relative frequency of broad-lined and narrow-lined active galactic nuclei-implications for unified 
schemes," Monthly Notices of the Royal Astronomical Society, vol. 252, pp. 586-592, 1991.

[150] A. Lamastra, G. C. Perola, and G. Matt, "A model for the X-ray absorption in compton-thin AGN," Astronomy and Astrophysics, vol. 449, no. 2, pp. 551-558, 2006.

[151] J. Fischer, E. Sturm, E. González-Alfonso et al., "HerschelPACS spectroscopic diagnostics of local ULIRGs: conditions and kinematics in Markarian 231," Astronomy and Astrophysics, vol. 518, no. 14676, article L36, 2010.

[152] C. Feruglio, R. Maiolino, E. Piconcelli et al., "Quasar feedback revealed by giant molecular outflows," Astronomy and Astrophysics, vol. 518, no. 13, article L155, 2010.

[153] D. S. N. Rupke and S. Veilleux, "Warm molecular hydrogen in the galactic wind of M82," The Astrophysical Journal, vol. 729, no. 2, article L27, 2011.

[154] E. Sturm, E. Gonzalez-Alfonso, S. Veilleux et al., "Massive molecular outflows and negative feedback in ULIRGs observed by Herschel-PACS," The Astrophysical Journal, vol. 733, no. 1, p. L16, 2011.

[155] M. Cano-Diaz, R. Maiolino, A. Marconi, H. Netzer, O. Shemmer, and G. Cresci, "Observational evidence of quasar feedback quenching star formation at high redshift," Astronomy \& Astrophysics, vol. 537, article L8, 2012.

[156] D. R. Ballantyne, Y. Shi, G. H. Rieke, J. L. Donley, C. Papovich, and J. R. Rigby, "Does the AGN unified model evolve with redshift? Using the X-ray background to predict the mid-infrared emission of AGNs," The Astrophysical Journal, vol. 653, no. 2, pp. 1070-1088, 2006.

[157] E. Treister and C. M. Urry, "The evolution of obscuration in active galactic nuclei," The Astrophysical Journal, vol. 652, pp. L79-L82, 2006.

[158] L. J. Tacconi, R. Genzel, R. Neri et al., "High molecular gas fractions in normal massive star-forming galaxies in the young Universe," Nature, vol. 463, no. 7282, pp. 781-784, 2010.

[159] E. Daddi, F. Bournaud, F. Walter et al., "Very high gas fractions and extended gas reservoirs in $\mathrm{z}=1.5$ disk galaxies," The Astrophysical Journal, vol. 713, no. 1, pp. 686-707, 2010.

[160] L. Shao, D. Lutz, R. Nordon et al., "Star formation in AGN hosts in GOODS-N," Astronomy and Astrophysics, vol. 518, no. 1, article L26, 2010.

[161] Y. Shi, G. H. Rieke, P. Ogle, L. Jiang, and A. M. DiamondStanic, "Cosmic evolution of star formation in type-1 quasar hosts since $\mathrm{z}=1$," The Astrophysical Journal, vol. 703, no. 1, pp. 1107-1122, 2009.

[162] C. Ricci, R. Walter, T. J.-L. Courvoisier, and S. Paltani, "Reflection in Seyfert galaxies and the unified model of AGN," Astronomy \& Astrophysics, vol. 532, article A102, 2011.

[163] A. Marconi, E. Oliva, P. P. van der Werf et al., "The elusive active nucleus of NGC 4945," Astronomy and Astrophysics, vol. 357, no. 1, pp. 24-36, 2000.

[164] R. Della Ceca, L. Ballo, F. Tavecchio et al., "An enshrouded active galactic nucleus in the merging starburst system Arp 299 revealed by BeppoSAX," The Astrophysical Journal, vol. 581, no. 1, pp. L9-L13, 2002.

[165] R. Maiolino, A. Comastri, R. Gilli et al., "Elusive active galactic nuclei," Monthly Notices of the Royal Astronomical Society, vol. 344, no. 4, pp. L59-L64, 2003.

[166] L. Ballo, V. Braito, R. Della Ceca, L. Maraschi, F. Tavecchio, and M. Dadina, "ARP 299: a second merging system with two active nuclei?” The Astrophysical Journal, vol. 600, no. 2, pp. 634-639, 2004.
[167] A. Caccianiga, P. Severgnini, R. Della Ceca, T. Maccacaro, F. J. Carrera, and M. J. Page, "Elusive AGN in the XMM-Newton bright serendipitous survey," Astronomy and Astrophysics, vol. 470, no. 2, pp. 557-570, 2007.

[168] M. Imanishi, R. Maiolino, and T. Nakagawa, "Spitzer infrared low-resolution spectroscopic study of buried active galactic nuclei in a complete sample of nearby ultraluminous infrared galaxies," The Astrophysical Journal, vol. 709, no. 2, pp. 801$815,2010$.

[169] M. Imanishi, C. C. Dudley, R. Maiolino, P. R. Maloney, T. Nakagawa, and G. Risaliti, "A Spitzer IRS low-resolution spectroscopic search for buried AGNs in nearby ultraluminous infrared galaxies: a constraint on geometry between energy sources and dust," Astrophysical Journal, Supplement Series, vol. 171, no. 1, pp. 77-100, 2007.

[170] M. Imanishi, "Luminous buried active galactic nuclei as a function of galaxy infrared luminosity revealed through spitzer low-resolution infrared spectroscopy," The Astrophysical Journal, vol. 694, no. 2, pp. 751-764, 2009.

[171] G. Risaliti, M. Imanishi, and E. Sani, "A quantitative determination of the AGN content in local ULIRGs through L-band spectroscopy," Monthly Notices of the Royal Astronomical Society, vol. 401, no. 1, pp. 197-203, 2010.

[172] E. Nardini, G. Risaliti, M. Salvati et al., "Exploring the active galactic nucleus and starburst content of local ultraluminous infrared galaxies through 5-8 $\mu \mathrm{m}$ spectroscopy," Monthly Notices of the Royal Astronomical Society, vol. 399, no. 3, pp. 1373-1402, 2009.

[173] E. Nardini, G. Risaliti, M. Salvati et al., "Spectral decomposition of starbursts and active galactic nuclei in $5-8 \mu \mathrm{m}$ Spitzer-IRS spectra of local ultraluminous infrared galaxies," Monthly Notices of the Royal Astronomical Society, vol. 385, no. 1, pp. L130-L134, 2008.

[174] D. A. Evans, W.-F. Fong, M. J. Hardcastle et al., "A Radio through X-Ray Study of the Jet/Companion-Galaxy Interaction in 3C 321," The Astrophysical Journal, vol. 675, pp. 10571066.

[175] A. D. Goulding and D. M. Alexander, "Towards a complete census of AGN in nearby Galaxies: a large population of optically unidentified AGN," Monthly Notices of the Royal Astronomical Society, vol. 398, no. 3, pp. 1165-1193, 2009.

[176] J. P. Perez-Beaupuits, H. W. W. Spoon, M. Spaans, and J. D. Smith, "The deeply obscured AGN of NGC 4945. I. SpitzerIRS maps of [Ne V], [Ne II], $\mathrm{H}_{2}$ 0-0 S(1), S(2), and other tracers," Astronomy \& Astrophysics, vol. 533, article A56, 2011.

[177] L. Armus, V. Charmandaris, J. Bernard-Salas et al., "Observations of ultraluminous infrared galaxies with the infrared spectrograph on the spitzer space telescope. II. The iras bright galaxy sample," The Astrophysical Journal, vol. 656, no. 1, pp. 148-167, 2007.

[178] Y. Ueda, S. Eguchi, Y. Terashima et al., "Suzaku observations of active galactic nuclei detected in the swift BAT survey: discovery of a "New Type" of buried supermassive black holes," The Astrophysical Journal, vol. 664, pp. L79-L82, 2007.

[179] K. Noguchi, Y. Terashima, and H. Awaki, "A new sample of buried active galactic nuclei selected from the second xmmnewton serendipitous source catalogue," The Astrophysical Journal, vol. 705, no. 1, pp. 454-467, 2009.

[180] L. Jiang, X. Fan, W. N. Brandt et al., "Dust-free quasars in the early Universe," Nature, vol. 464, no. 7287, pp. 380-383, 2010 . 
[181] H. Hao, M. Elvis, F. Civano et al., "Hot-dust-poor type 1 active galactic nuclei in the COSMOS survey," The Astrophysical Journal, vol. 724, no. 1, pp. L59-L63, 2010.

[182] H. Hao, M. Elvis, F. Civano, and A. Lawrence, "Hot-dustpoor quasars in mid-infrared and optically selected samples," The Astrophysical Journal, vol. 733, no. 2, article 108, 2011. 

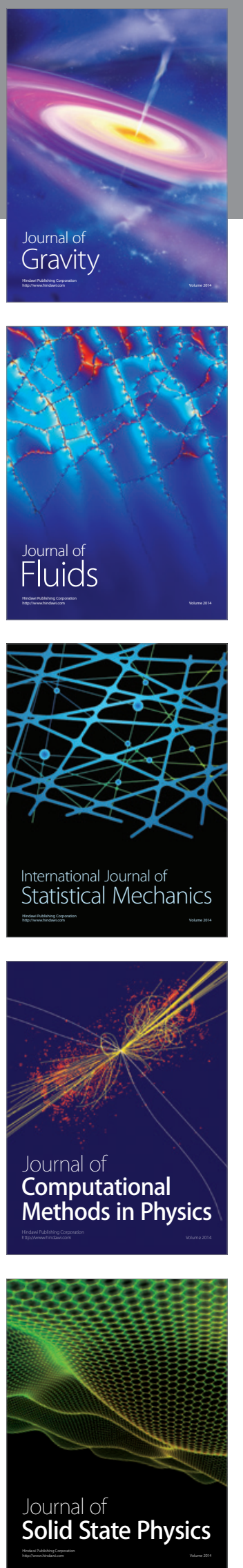

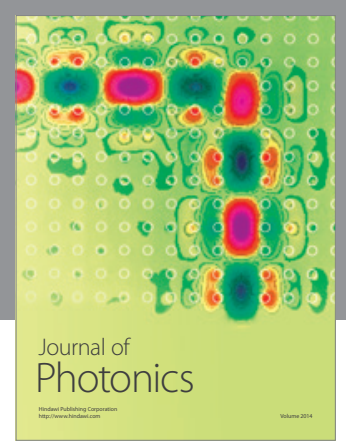

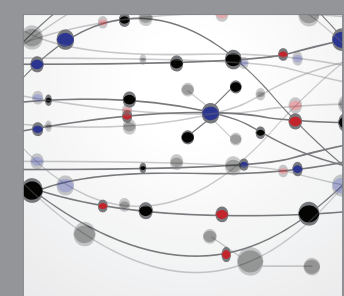

The Scientific World Journal
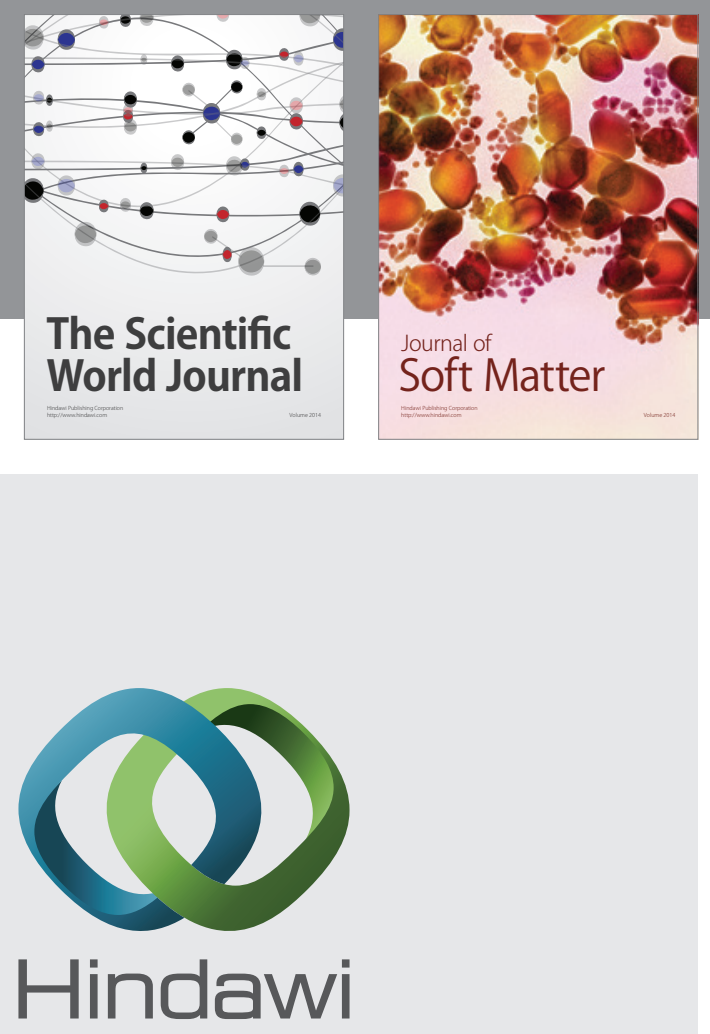

Submit your manuscripts at

http://www.hindawi.com
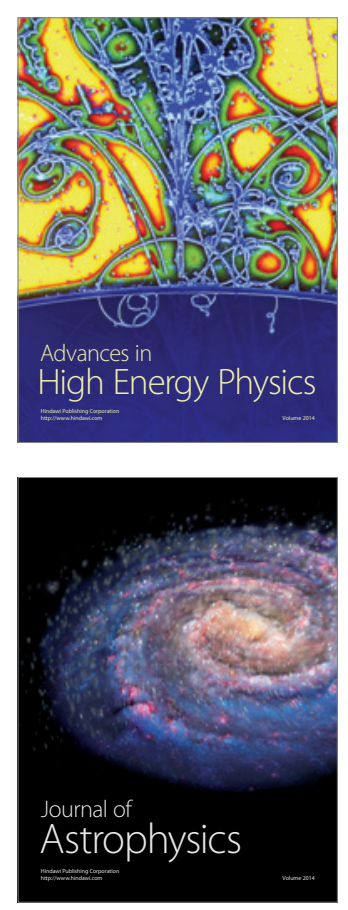
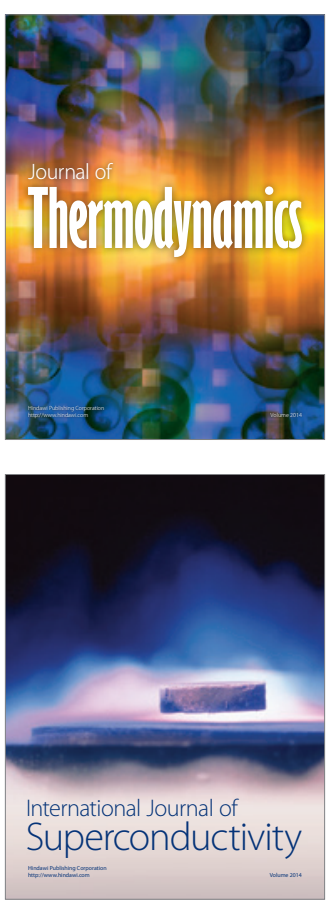
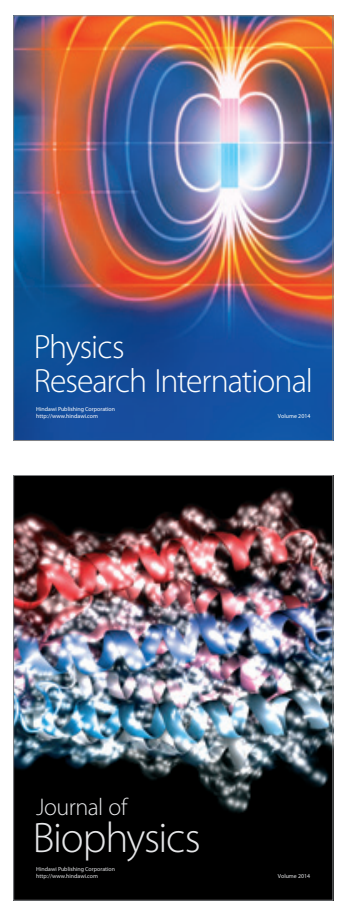
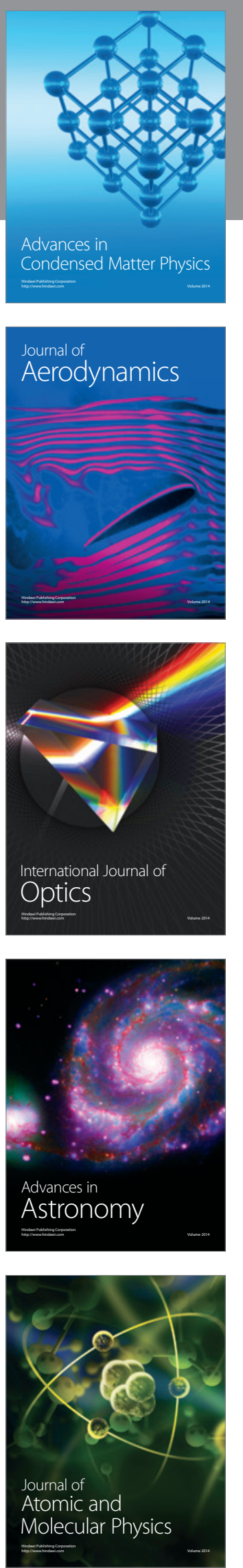